\title{
Differential Processing of Semicircular Canal Signals in the Vestibulo-Ocular Reflex
}

\author{
Dora E. Angelaki, ${ }^{1}$ Bernhard J. M. Hess, ${ }^{2}$ and Jun-Ichi Suzuki ${ }^{3}$ \\ 'Department of Surgery (Otolaryngology), University of Mississippi Medical Center, Jackson, Mississippi 39216- \\ 4505, ${ }^{2}$ Department of Neurology, University Hospital Zurich, $\mathrm{CH}-8091$, Switzerland, and ${ }^{3}$ Department of \\ Otolaryngology, Teikyo University, Tokyo, Japan
}

\begin{abstract}
Selective semicircular canal inactivation and three-dimensional eye movement recordings have been used to investigate the spatial organization of vestibular signals in the vestibulo-ocular reflex (VOR) of rhesus monkeys. In animals with one pair of semicircular canals inactivated, afferent activity no longer codes all spatial components of head angular velocity. If it were the activation pattern of semicircular canal afferents alone that determines VOR slow phase eye velocity, the head velocity components along the sensitivity vectors of the remaining intact semicircular canals would determine the orientation of slow phase eye velocity. Thus, angular head velocity and slow phase eye velocity would not necessarily always align. Alternatively, if vestibulo-ocular signals coded absolute angular head motion in space based on both semicircular canal and otolith afferent information, one might expect a spatial transformation of the encoded head angular velocity signals such that slow phase eye velocity and angular head velocity continue to spatially align.
\end{abstract}

Examination of the VOR at different frequencies between $0.01 \mathrm{~Hz}$ and $1 \mathrm{~Hz}$ revealed a frequency-specific spatial organization of vestibulo-ocular signals. Mid and high frequency vestibulo-ocular responses were determined exclusively by the orientation of the sensitivity vectors of the remaining intact semicircular canals. In contrast, low frequency vestibulo-ocular responses were largely determined by the orientation of the head relative to gravity. These low frequency responses after selective semicircular canal inactivation could be predicted and simulated by a simple model where semicircular canal signals are spatially transformed from a head-fixed to a space-fixed (inertial) representation of angular head velocity. These findings suggest that low frequency vestibulo-ocular responses are dominated by inertial vestibular signals that detect absolute head motion in space based on both semicircular canal and otolith afferent information. Inertial vestibular signals are likely to contribute to head control and motor coordination of gaze, head and body posture.

\footnotetext{
Received Feb. 28, 1995; revised June 23, 1995; accepted June 28, 1995.

This work was supported by grants from the NIH (EY 10851) and the Swiss National Science Foundation (\#31-32484.91). Experiments were performed at the Vestibulo-ocular Laboratory. Department of Neurology, University of Zürich.

Correspondence should be addressed to Dora Angelaki, Department of Surgery (Otolaryngoloyy), University of Mississippi Medical Center. 2500 North State Street, Jackson. MS 39216-4505.

Copyright (c) 1995 Society for Neuroscience $0270-6474 / 95 / 157201-16 \$ 05.00 / 0$
}

[Key words: eye movements, vestibular, vestibulo-ocular, posture, gaze, spatial orientation, gravity, neural computation, rhesus monkeys]

The vestibulo-ocular reflex (VOR) is optimally suited to reduce retinal image slip during high frequency head movements when visual mechanisms are insufficient for gaze stabilization. It uses semicircular canal afferent signals that encode head angular velocity with high fidelity in the mid and high frequency range of rotatory head movements. Due to peripheral mechanical dynamics, however, semicircular canal afferents carry less precise head angular velocity signals during low frequency head rotations. Below approximately $0.1 \mathrm{H} \iota$ the sensitivity of primary semicircular canal afferents decreases and the phase angle begins to lead head velocity, reaching values of $70-90^{\circ}$ at $0.01 \mathrm{~Hz}$ in monkeys (Fernandez and Goldberg, 1971). Despite this limitation in head velocity transduction, the VOR exhibits an extended low frequency performance that outranges the frequency bandwidth of velocity sensitivity of semicircular canal afferents.

It has been proposed that the enhanced low frequency VOR dynamics is due to a mathematical integration of semicircular canal primary afferent activity by a central ncural nctwork, a process called "velocity storage." According to this proposal, this network constitutes a parallel loop in VOR circuitry which basically carries the same vestibular information as the classical, short-latency VOR pathways, however, low pass filtered and extended towards lower frequencies (Cohen et al., 1977; Raphan et al., 1979). The existence of powerful visual following and ocular pursuit mechanisms that are optimally tuned to stabilize gaze at low frequencies seems, however, to eliminate the need for such additional vestibular low frequency stabilization mechanism.

Recent observations on low frequency vestibulo-ocular response characteristics have cast further doubts on the interpretation of the velocity storage as an oculomotor mechanism whose primary function would simply be to extend the low frequency bandwidth of the VOR by perseverating semicircular canal afferent inputs (Solonon and Cohen, 1992; Angelaki and Hess, 1994, 1995). In an alternative view, the velocity storage is considered as part of a more global vestibulo-motor system which can best be characterized as an inertial vestibular system that codes for head and body motion in space (Angelaki and Hess, 1994, 1995). This system utilizes both semicircular canal afferent signals which detect the angular velocity of the head independently of its orientation in space and otolith afferent information which codes instantaneous head orientation relative to 
A Postrotatory VOR before head tilt

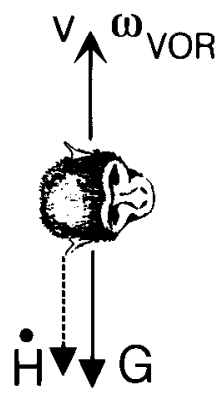

B VOR with intact SCC

Figure 1. Spatial transformation of head-fixed semicircular canal signals (v) to space-referenced inertial VOR signals $\left(\omega_{\text {VoR }}\right) . A$, Vector orientations of head velocity and VOR slow phase velocity without (left) and after a head tilt (right). B, Vector orientations of head velocity and VOR slow phase velocity in animals with intact semicircular canals (SCC) (left) and in animals with the right anterior/left posterior canal inactivated (right). $\mathbf{H}$, Head angular velocity vector; $\mathbf{G}$, gravity vector.

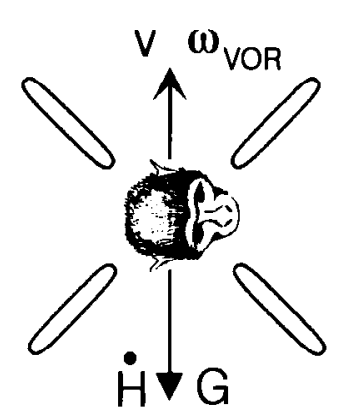

after head tilt

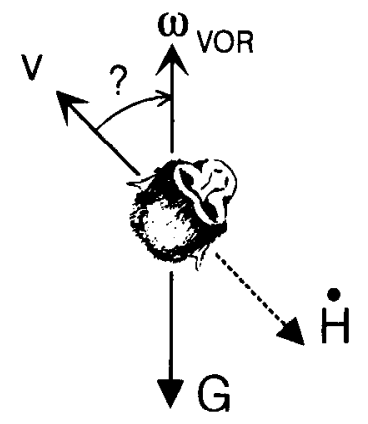

after inactivation of RALP SCC

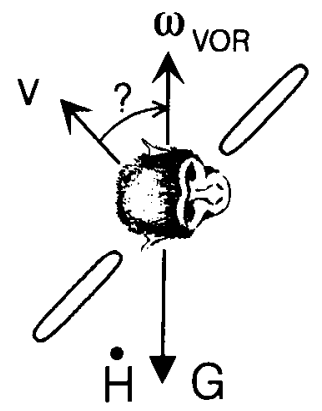

gravity. Combining these signals enables the inertial vestibular system to detect absolute motion in space.

To experimentally verify this proposal semicircular canal and otolith afferent activity must be spatially segregated. In most experimental studies of the VOR, semicircular canals are activated during earth-vertical axis rotations such that the angular head velocity $(\dot{\mathbf{H}})$ and the direction of gravity $(\mathbf{G})$ always align. Under this condition, central vestibular signals coding head angular velocity in space $\left(\omega_{\mathrm{vOR}}\right)$ are indistinguishable from semicircular canal afferent signals coding angular head velocity in a head-fixed fashion (v) (Fig. 1A, left). To investigate whether there are inertial vestibular signals coding head motion in space, semicircular canal afferent activity must be spatially dissociated from otolithic signals coding head orientation relative to gravity. One way to distinguish semicircular canal signals from inertial vestibular signals is to apply fast, short-lasting head tilts during a postrotatory vestibulo-ocular response (Fig. 1A, right). After such head tilt, semicircular canal afferents continue to reflect the preceding angular head motion (i.e., vector $\mathbf{v}$ is opposite to $\dot{\mathbf{H}}$ and its orientation does not change relative to the head), whereas otolith afferents detect a different orientation of the head relative to gravity (along vector $\mathbf{G}$ ). If inertial vestibular signals are centrally computed and relayed to the VOR, the elicited eye velocity vector would not align with $\mathbf{v}$ but rather with $\mathbf{G}$. Using this approach, we have found that slow phase eye velocity in the late postrotatory VOR responses aligned with gravity while the initial slow phase velocity remained invariant relative to the head (Angelaki and Hess, 1994, 1995). Thus, the late postrotatory VOR responses seem to represent head velocity in space $\left(\omega_{\mathrm{VOR}}\right)$, consistent with the concept of an inertial (i.e., spacefixed) representation of head velocity. Interestingly, postrotatory realignment of slow phase velocity with gravity was found irrespectively of the plane of head rotation and tilt. Thus, spatial transformation of semicircular canal signals seems to characterize the central processing of not only lateral (Harris, 1987; An- gelaki and Anderson, 1991; Merfeld et al., 1991, 1993) but also vertical semicircular canal activity (Angelaki and Hess, 1994, 1995).

The results obtained by this type of experiments have to be interpreted, however, with some caution. The transient head tilts also stimulate in-plane semicircular canal pathways that could interfere with the spatial processing of postrotatory signals. The timing of tilt relative to the stop of the previous head rotation, as well as tilt duration are further parameters that could possibly influence the spatio-temporal transformation of postrotatory responses. For example, head tilts at slow velocity taking 4-6 sec to complete did no longer elicit transformation of vertical or torsional postrotatory responses towards alignment with gravity (Angelaki and Hess, 1994). Such difficulties, as well as previous reports that vertical optokinetic afternystagmus does not reorient relative to gravity have been taken as arguments against the existence of inertial vestibular signals (Dai et al., 1991; Raphan et al., 1994).

The existence of an inertial vestibular system and its effect on VOR slow phase eye velocity can be studied without recourse to transient head tilts. Here, we report the effects of dissociating a head-fixed from a space-referenced inertial representation of head angular velocity in the VOR as a result of selective semicircular canal inactivation (Fig. $1 B$ ). After selective inactivation of a coplanar pair of semicircular canals, the imposed head velocity vector (iI) and the vector $\mathbf{v}$ of scmicircular canal afferent activity are not necessarily aligned due to the missing information from the inactivated semicircular canals. Primary otolith afferent activity, on the other hand, is not affected by canal inactivation and thus encodes the correct spatial orientation of the head movement relative to gravity. During rotation in eardown position of an animal with inactivated right anterior/left posterior canals, for example, the semicircular canal activity vector $\mathbf{v}$ would be aligned with the remaining intact left anterior/ 


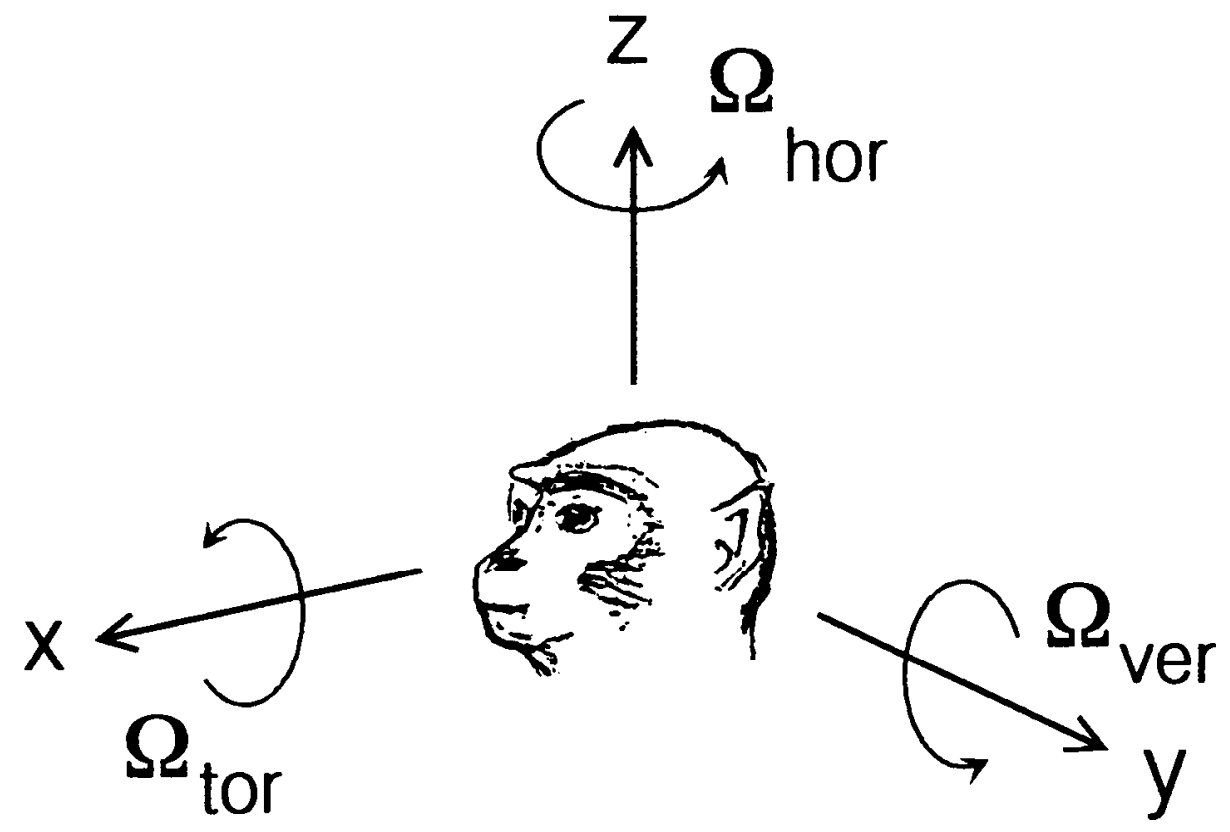

Figure 2. Head-fixed, right-handed coordinate system used to parameterize eye position and velocity. Positive directions of ocular rotation are designated by the arrows. $\Omega=\left(\Omega_{\text {tur }}, \Omega_{\text {ver }}\right.$, $\left.\Omega_{\text {hor }}\right)$ : angular velocity vector relative to head-fixed coordinates $x$ (along nasooccipital axis), y (along interaural axis), and $z$ (along vertical axis). The $\mathrm{x}-\mathrm{y}$ plane is tilted $15^{\circ}$ up relative to stereotaxic horizontal.

right posterior canal sensitivity vector ${ }^{1}$ and not be parallel to the imposed head velocity vector $\mathbf{H}$. If vestibular signals in the VOR are head-fixed and determined exclusively by the semicircular canal afferent activity, the remaining intact semicircular canals should determine the spatial orientation of VOR slow phase eye velocity. Alternatively, if inertial vestibular signals do exist and contribute to the VOR, slow-phase eye velocity would not be solcly dependent on the pattern of semicircular canal activation but also on head orientation relative to gravity. We find that the spatio-temporal characteristics of mid and high frequency VOR solely depends on semicircular canal activity. Inertial vestibular signals representing head velocity in space, on the other hand, determine the low frequency VOR responses. Thus, low frequency VOR responses in rhesus monkeys reflect the contribution of an inertial vestibular system that spatially transforms semicircular canal afferent activity to code angular velocity of the head in space.

\section{Materials and Methods}

Animal preparation and eye movement recording. In four rhesus monkeys, semicircular canals were inactivated by drilling a small hole in the bony canal (away from the sensory epithelia), cutting the membranous semicircular canal duct with the tip of a sharp knife, and filling the hole with bony chips, as previously described (Ewald, 1892; Money and Scott, 1962; Suzuki et al., 1991). In two animals, the lateral canals were plugged bilaterally and in one animal, the right anterior and left posterior canals were plugged. In the fourth animal, all but the left posterior canal were plugged. All animals were chronically prepared with skull bolts to restrain the head during experiments and implanted with a dual search coil for three-dimensional eye movement recording using the magnetic search coil technique (details of these procedures are provided in Angelaki and Hess, 1994). Operation procedures and animal treatment were in accordance with the NIH guidelines for the care and use of laboratory animals. All surgeries were performed under intubation anaesthesia with an $\mathrm{O}_{2}-\mathrm{N}_{2} \mathrm{O}$ mixture supplemented by halothane as required to maintain a deep, constant level of anaesthesia. To allow time for recovery, successive surgeries were separated by at least 2-3 weeks. For the eye coil and head implant surgeries, a single shot of a long-lasting broad spectrum antibiotic (Bicillin A) was adminis-

'The left anterior/right posterior canal sensitivity vector is defined as a unity vector perpendicular to the plane of the canals pointing in the direction of activivation of the anterior semicircular canal (vector $v$ in Fig. $1 B$, right). trated at the time of operation. In addition, an analgesic (Voltaren) was also administered during the first few days after the operation. For the plugging surgeries, antibiotics (Rocephin) combined with corticosteroids (Ultracorten) were daily administered during the first postoperative week. After the plugging operation (which was usually completed by mid-day), animals were allowed to recover in a completely darkened room with amplc access to food and water. For the next 12-15 hr the operated animals were periodically checked by personnel using infrared goggles. Other than a high-frequency head instability in the plane of the plugged canals which persisted for approximately 1-2 d after surgery, animals had behaviorally recovered by mid morning the following day when most of the data presented in this study were collected (see below).

Prior to experimental sessions, animals were trained to fixate small target lights for fluid reward (for details, see Angelaki and Hess. 1994. 1995). Calibration of the coil signals was performed in two stages, one prior to implantation to determine the coil sensitivity vectors and the second after coil implantation (Hess et al., 1992). The in vivo calibration was routinely repeated daily prior to each experimental protocol. Calibration parameters remained constant on different experimental days such that the same calibration values could be used on several successive experiments. Since animals were not water-deprived for at least $1-$ 2 weeks after each operation, data collected on the first postoperative day after semicircular canal plugging were calibrated based on calibration files obtained 3-4 d before the operation. Spontaneous eye movements in the light and Listing's plane served as a control for the adequacy of use of previous calibration files: Listing's plane during spontaneous eye movements in upright position continued to be thin with negligible offsets and stable orientation relative to the head.

Three-dimensional eye position was sampled at $833 \mathrm{~Hz}$ and subsequently expressed in head-fixed Cartesian coordinates as rotation vectors (E) (Haustein, 1989). In this description, a given eye position is described as a three-component vector whose direction is defined by the axis of rotation between a reference position and current eye position and its magnitude as the tangent of half the rotation angle about this axis. The eye angular velocity vector, $\Omega$ was computed from the eye position vector, $\mathbf{E}$, as $\Omega=2(d \mathbf{E} / d t+\mathbf{E} \times d \mathbf{E} / d t) /\left(1+|\mathbf{E}|^{2}\right)$.

Eye position $\left(E_{\mathrm{tor}}, E_{\mathrm{ver}}, E_{\mathrm{hor}}\right)$ and angular velocity vectors $\left(\Omega_{\mathrm{tor}}, \Omega_{\mathrm{ver}}\right.$, $\Omega_{\text {hor }}$ ) were expressed relative to a right-handed, head-fixed coordinate system defined by the naso-occipital ( $x$-axis, torsional component), the interaural (y-axis, vertical component) and the vertical ( $z$-axis, horizontal) head axes. Positive directions were defined as counterclockwise (i.e., rotation of the upper pole of the eye towards the right ear), downward and leftward, respectively (Fig. 2). Examination of gaze holding and spontaneous eye movements in the light revealed no signs of a deficiency in the velocity to position integrator. In addition, other than a down-beating vertical nystagmus which occurs often in rhesus mon- 

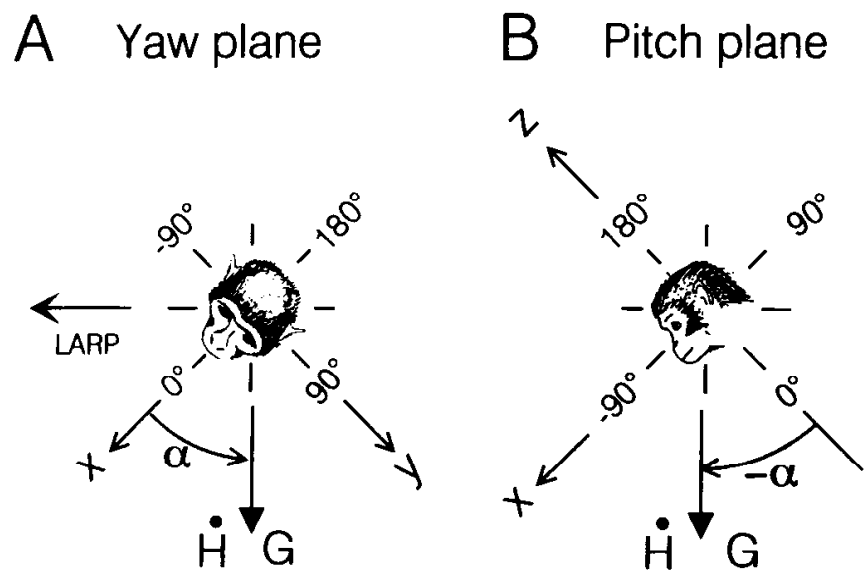

Figure 3. Spatial orientation of the head velocity $(\dot{\mathbf{H}})$ and gravity $(\mathbf{G})$ vectors in the head-fixed, right-handed coordinate systcm. $A$, Vector orientation in the yaw plane. An angle of $0^{\circ}$ between the positive $\mathbf{x}$-axis and gravity $\mathbf{G}$ corresponds to prone position. $B$, Vector orientation in the pitch plane. An angle of $0^{\circ}$ between the negative $z$-axis and gravity $\mathbf{G}$ corresponds to the upright position. Other orientations in either plane are defined by the polar angle $\alpha$. The left anterior/right posterior canal sensitivity vector $(L A R P)$ is oriented at $\alpha=-45^{\circ}$.

keys, spontaneous nystagmus did not significantly increase after the plugging operations. Accordingly, eye angular velocity was further processed in order to remove the fast phases of nystagmus based on time and amplitude windows set for the second derivative of the magnitude of the eye velocity vector.

Experimental protocols and data analysis. During the experiments, the monkeys were seated in a primate chair with their heads restrained in a position such that the horizontal stereotaxic plane was tilted $15^{\circ}$ nose-down. This head position was used in order to place the vertical semicircular canals approximately perpendicular and the lateral semicircular canals approximately parallel to an earth-horizontal plane. In all experimental protocols the axis of rotation was always earth-vertical. Thus, any dynamic contribution of the otolith system to the VOR was eliminated. The position of the animals relative to the axis of rotation changed such that different combinations of horizontal, vertical, and torsional eye movements could be elicited. Head rotation and semicircular canal activation were expressed in terms of velocity vectors rather than the more traditional description in terms of rotation planes. Accordingly, a given head gotation is described by the associated head velocity stimulus vector $\mathbf{H}$ that is normal to the plane of rotation according to the right hand rule. For example, yaw rotation is described by a vector along the vertical head axis. Similarly, pitch rotation is described by a vector along the interaural axis and roll rotation by a vector along the nasooccipital axis.

As the position of the animals relative to the axis of rotation was systematically, changed in a given experimental protocol, the head velocity vector $\mathbf{H}$ (and $\mathbf{G}$ ) changed orientation relative to the head. The orientation of vectors $\mathbf{H}$ and $\mathbf{G}$ was also expressed relative to the coordinate system of Figure 2 in terms of a polar angle $\alpha$ defined according to the right-hand rule. For the yaw plane, for example, a stimulus angle of $0^{\circ}\left(180^{\circ}\right)$ described a rotation in prone (supine) position and a stimulus angle of $\pm 90^{\circ}$ described a rotation in ear-down positions (Fig. $3 A$ ). For the pitch plane, an angle of $0^{\circ}$ corresponded to a rotation in upright position, whereas an angle of $90^{\circ}$ or $-90^{\circ}$ corresponded to rotation in supine or prone position, respectively (Fig. $3 B$ ). The spatial orientation of the respective VOR velocity vectors was described in a similar fashion. For example, the orientation of a VOR velocity vector in the yaw plane was also described by an angle $\alpha$ relative to the positive $x$-axis. Experimentally, this angle was estimated as the inverse tangent of the ratio of vertical and torsional peak eye movement. Thus, an angle of $0^{\circ}$ or $180^{\circ}$ defined a pure torsional eye movement. Similarly, an angle of $90^{\circ}$ defined a pure vertical eye velocity. Amplitudes were considered positive when response phases were between $-90^{\circ}$ and $90^{\circ}$ and negative otherwise. The three-dimensional organization of the VOR before and after selective semicircular canal inactivation was studied by applying long-duration steps of angular velocity and sinusoidal oscillations at seven frequencies: $0.01,0.02,0.05,0.1,0.2,0.5$, and $1 \mathrm{~Hz}$.

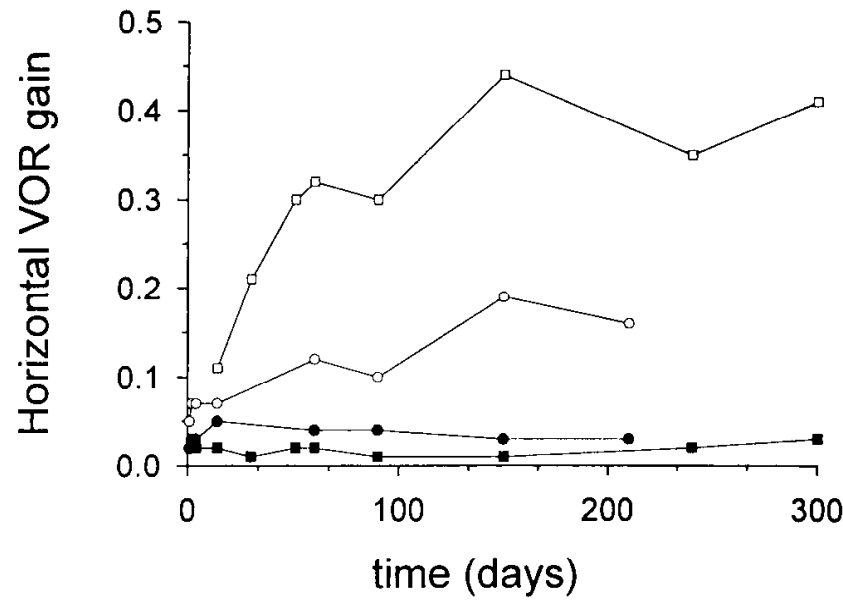

Figure 4. Horizontal VOR gain as a function of time after bilateral surgical inactivation of the lateral canals in two animals. Stimulus frequency: $0.5 \mathrm{~Hz}$ (open symbols); $0.01 \mathrm{~Hz}$ (solid symbols).

For the sinusoidal protocols, peak amplitude was constant at $58 \% \mathrm{sec}$, except at the highest frequency $(1 \mathrm{~Hz})$, where peak amplitude was reduced to $35 \%$ sec. Gain and phase were computed for each stimulus cycle (usually 10 at high frequencies and three or six at $0.01 \mathrm{~Hz}$ and $0.02 \mathrm{~Hz}$, respectively) by fitting a sine wave function to stimulus velocity and to each component of the desaccaded eye velocity vector using a nonlinear least squares algorithm based on the I evenherg-Marquardt method. For both intact and canal plugged animals, the gain of each response component (horizontal, vertical, and torsional) was expressed as the ratio of response amplitude and peak head velocity. Data reported here are based on median values. A phase of zero describes an eye velocity response that is perfectly compensatory to head velocity.

The majority of the dala presented here were collected the first day after inactivation of selective semicircular canals. To avoid adaptation, animals were kept in a completely darkened room after surgery until the following morning at which time they were tested in the laboratory. Care was taken not to expose animals to anything but a dim light necessary for placing the animals into the monkey chair. Experimental testing in darkness lasted about four hours. In between trials, animals were given periods of rest, most of which in darkness, even though brief periods of light were also delivered approximately every half hour. As mentioned above, animals had completely recovered from the inhalation anesthesia during the period of about $20 \mathrm{hr}$ which elapsed between completion of surgery and experimental testing. To maintain a constant level of alertness, a small dose of $d$-amphetamine (1.5 mg orally or 1 mg i.m.) was administered prior to experimental testing. After testing, animals were returned to their home cage where they lived in a social group of three to five animals with the normal $12 \mathrm{hr}$ of dark and light cycle. Most data presented here were recorded during the first day after surgery before animals were exposed to a normal visual environment. We have assumed that VOR adaptation was minimal without normal visuo-motor experience. There was substantial recovery of VOR in these animals over time (Angelaki et al., 1994). This adaptation was, however, limited to mid and high frequencies as illustrated in Figure 4 (open symbols, $0.5 \mathrm{~Hz}$; solid symbols, $0.01 \mathrm{~Hz}$ ). Due to the ahsence of any VOR recovery during $0.01 \mathrm{~Hz}$ oscillations, data acquired on subsequent weeks after the operation have also been included here (see Figs. 15-17).

Model simulations. The spatial organization of space-fixed vestibuloocular signals was modeled in terms of a simple geometrical transformation (Fig. 5). Accordingly the vertical and lateral canal activity vectors (represented by $\mathbf{v}_{\mathrm{vc}}$ and $\mathbf{v}_{1 \mathrm{C}}$, respectively, in Fig. $5 A, B$ ) correspond to the components of the head-fixed semicircular canal afferent vector v (e.g., Figs. 1, 5C). The geometric transformations used in the model are based on previous experimental results regarding the spatial organization of the late postrotatory responses after head and body tilts (Angelaki and Hess, 1994, 1995). Based on these studies, the spatial transformation $T_{G}$ could best be described by two different mechanisms.

(1) The vertical semicircular canal signals are spatially transformed by a simple projection of the vertical canal activation vector $\mathbf{v}_{\mathrm{vc}}$ onto the gravity vector $\mathbf{G}$. In an animal with inactivated right anterior/left 
A
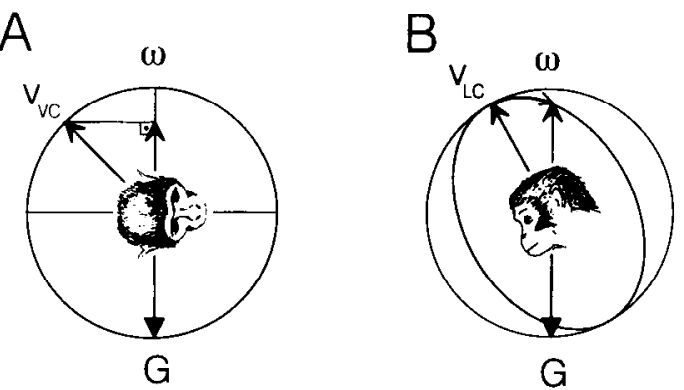

C

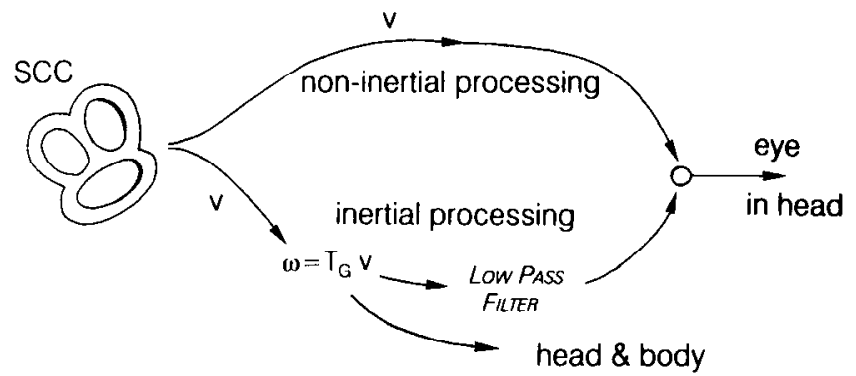

Figure 5. Spatial transformation of head-fixed semicircular canal signals $(v)$ to space-referenced inertial vestibular signals $(\boldsymbol{\omega})$. $A$, Vertical canal activity $\left(\mathbf{v}_{\mathrm{vc}}\right)$ is spatially transformed by projection of the activation vector onto a line parallel to gravity. $B$, Lateral canal activity $\left(v_{\mathrm{LC}}\right)$ is spatially transformed by a combination of vector rotation plus contraction. Thus, rotated vectors outline an elliptic instead of a circular contour. $C$, Schematic diagram of two main streams of central vestibular processing of semicircular canal signals $(S C C)$ relative to noninertial head-fixed and space-fixed inertial reference systems. $\mathbf{v}$, Semicircular canal activity vector; $\omega$, transformed space-fixed vector; $T_{G}$, gravitydependent spatial transformation (rotation-contraction or projection).

posterior canals, for example, the space-referenced velocity vector $\omega$ would be generated by projection of the afferent activity vector $\mathbf{v}_{\mathrm{vC}}$ (in this case $\mathbf{v}_{\mathrm{vc}}$ is parallel to the LARP sensitivity vector) onto the direction of gravity $\mathbf{G}$ (Fig. $5 A$ ). This projection transformation can be described by trigonometric equations that are presented in the Results separately for the yaw and pitch planes (see Figs. 14, 17).

(2) The lateral semicircular canal signals, on the other hand, are spatially transformed by a process that can best be described as a combination of rotation plus contraction (i.e., amplitude reduction, Fig. $5 B$; for a more detailed description of these mathematical transformations, see Angelaki and Hess, 1995). Specifically, the space-referenced vector $\omega$ is generated by "shrinking" and simultaneously rotating vector $\mathbf{v}_{\mathrm{LC}}$ towards the direction of gravity. This transformation can be mathematically represented by the equation

$$
\omega=T_{G} \mathbf{v}_{\mathrm{LC}}=c(\alpha) R(\alpha) \mathbf{v}_{\mathrm{LC}},
$$

where $R(\alpha)$ is a rotation operator, $c(\alpha)$ is a contraction factor, and $\alpha$ is the angle between the lateral canal activity vector $v_{1 . C}$ and gravity $\mathbf{G}$ (i.e., the angle of head tilt from upright, e.g., Fig. $3 B$ ). For a rotation about the pitch axis, for example, $R(\alpha)$ can be expressed as

$$
R(\alpha)=\left(\begin{array}{ccc}
\cos (\alpha) & 0 & -\sin (\alpha) \\
0 & 1 & 0 \\
\sin (\alpha) & 0 & \cos (\alpha)
\end{array}\right)
$$

The contraction of angular velocity from a circular onto an elliptical envelope can be described as $(\lambda<1)$

$$
c(\alpha)=\frac{\lambda}{\sqrt{\lambda^{2} \cos ^{2}(\alpha)+\sin ^{2}(\alpha)}} .
$$

Note that $c(\alpha)=1$ for $\lambda=1$ (no contraction). In accordance with previous model simulations of postrotatory nystagmus, we used $\lambda=$ 0.3 (Angelaki and Hess, 1995). Application of this contraction factor $c(\alpha)$ in Equation 1 results in a maximal amplitude reduction of $70 \%$ for a rotation through an angle of $\alpha= \pm 90^{\circ}$.
Spatially transformed semicircular canal signals are subsequently low-pass filtered before contributing to slow phase eye velocity (Fig. $5 C$ ). It should be noted that the model simulations presented here apply to a spatial analysis of data acquired from steady-state sinusoidal responses. Thus, no dynamics had to be included to compute the spatial organization of slow phase velocity. The simulations have been restricted to either a head-fixed or a space-fixed representation (rather than a combination of both). It will be shown, however, that the vestibuloocular reflex seems to include both head-fixed and space-fixed vestibular signals at different frequency bandwidths (according to Fig. 5C). To avoid complexity, the quantitative analysis focuses on three stimulus frequencies where head-fixed and inertial vestibular signals are well segregated, namely: $1.14 \mathrm{~Hz}$ and $0.21 \mathrm{~Hz}$ (both frequencies outside the "velocity storage" influence) and $0.01 \mathrm{~Hz}$ (at which frequency the velocity storage is clearly dominating VOR slow phase velocity).

In the quantitative analyses and model simulations, three simplifying assumptions have been made: (1) The synergistic vertical canals on the two sides of the head have been represented by a single activation plane oriented at an angle of $45^{\circ}$ from the naso-occipital and interaural axes. Accordingly, the LARP (left anterior/right posterior) and RALP (right anterior/left posterior) sensitivity vectors were defined by a clockwise $45^{\circ}$ rotation of the $\mathrm{x}$ - and $\mathrm{y}$-axes, respectively. Similarly, the lateral canal sensitivity vector was assumed to be parallel to the animal's z-axis (z-axis formed an angle of $15^{\circ}$ with a line perpendicular to the horizontal stereotaxic plane). (2) The gain of the torsional and vertical response components have been assumed to be equal. (3) The system has been assumed to be linear. Our main findings in terms of a head-fixed versus a space-fixed representation of head angular velocity depended little on a more precise evaluation of the parameters in assumptions 1 and 2 .

\section{Results}

\section{Frequency-dependent VOR spatial organization}

After inactivation of the right anterior and left posterior semicircular canals, rotation about the animal's RALP axis resulted in negligible vertical and torsional nystagmus. Examples of the torsional, vertical and horizontal slow phase eye velocity elicited by a step change in head velocity from zero to $90 \% \mathrm{sec}$ are shown in Figure $6 \mathrm{~A}$. To stimulate the right anterior/left posterior canals, the monkey was rotated about an earth-vertical axis in a position halfway between right ear-down and supine; that is, the axis of rotation was parallel to a line in the yaw plane intersecting the angle between the negative $\mathrm{x}$ - and $\mathrm{y}$-axes at $45^{\circ}$ (see sketch of monkey head in Fig. 6A). To maximally stimulate the left anterior/right posterior canals, the animal was positioned perpendicular to the former orientation such that the axis of rotation was parallel to a line in the head yaw plane intersecting the angle between the negative $x$ - and positive $y$-axes at $45^{\circ}$ (Fig. $6 B$ ). During rotation about the LARP axis, both torsional and vertical eye movements of approximately equal amplitude were elicited, each component decaying to zero with similar time courses. The resulting slow phase velocity trajectory in the yaw plane was a straight line parallel to the LARP axis, as apparent when plotting the vertical versus the torsional response component (Fig. $6 B$, right).

Because the right anterior/left posterior canals were inactivated, a combination of vertical and torsional eye movements were also elicited during rotation about the roll and pitch axes (Fig. $6 C, D)$. In intact animals this stimulation elicits a pure torsional or vertical response, respectively, due to symmetric activation of both vertical canal pairs. The vertical and torsional components elicited after plugging in both supine and ear-down positions differed only in the apparent time constant of decay. The time constant of the torsional component was longer compared to that of the vertical component during rotation in supine position. Similarly, the time constant of the vertical response component was longer compared to that of the torsional response 
A Rotation in Ralp plane
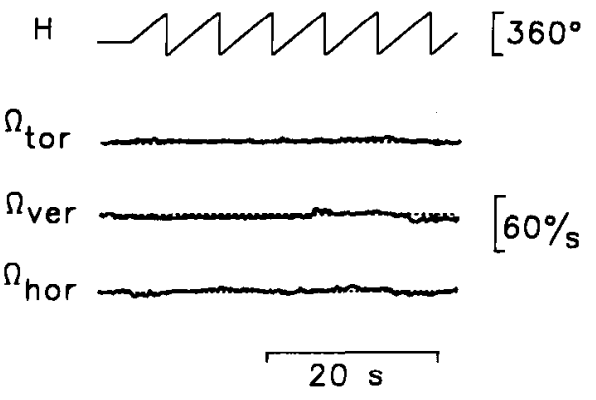

B Rotation in Larp plane H WWMMMMM [360

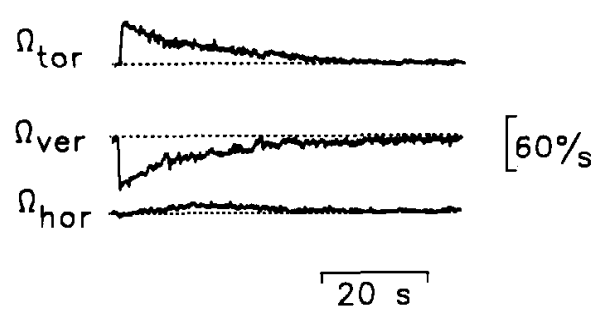

C Rotation in Roll plane ${ }^{\circ} M M M M M M M\left[360^{\circ}\right.$

Figure 6. VOR step responses as a function of head orientation relative to gravity 2 weeks after inactivation of the right anterior/left posterior semicircular canals. $A$, Rotation in the right anterior/ left posterior canal plane (RALP). $B$ Rotation in the left anterior/right posterior canal plane (LARP). $C$, Rotation in the roll plane (supine position). $D$, Rotation in the pitch plane (left eardown position). Torsional $\left(\Omega_{\text {tor }}\right)$, vertical $\left(\Omega_{\mathrm{vcr}}\right)$, and horizontal $\left(\Omega_{\mathrm{hor}}\right)$ slow phase eye velocity responses elicited by a step change in angular head velocity from zero to $90^{\circ} / \mathrm{sec}$. Time constants of the torsional/vertical component: $15.3 \mathrm{sec} / 21.4 \mathrm{sec}(B), 16.3 \mathrm{sec} /$ $4.6 \mathrm{sec}(C)$, and $4.9 \mathrm{sec} / 7.3 \mathrm{sec}(D)$ (cstimated as the time interval in which velocity dropped to $1 / e$ of its maximal value). Dotted lines mark zero slow phase eye velocity (left) and the direction of slow phase velocity for pure torsional, vertical, RALP and LARP VOR (right).
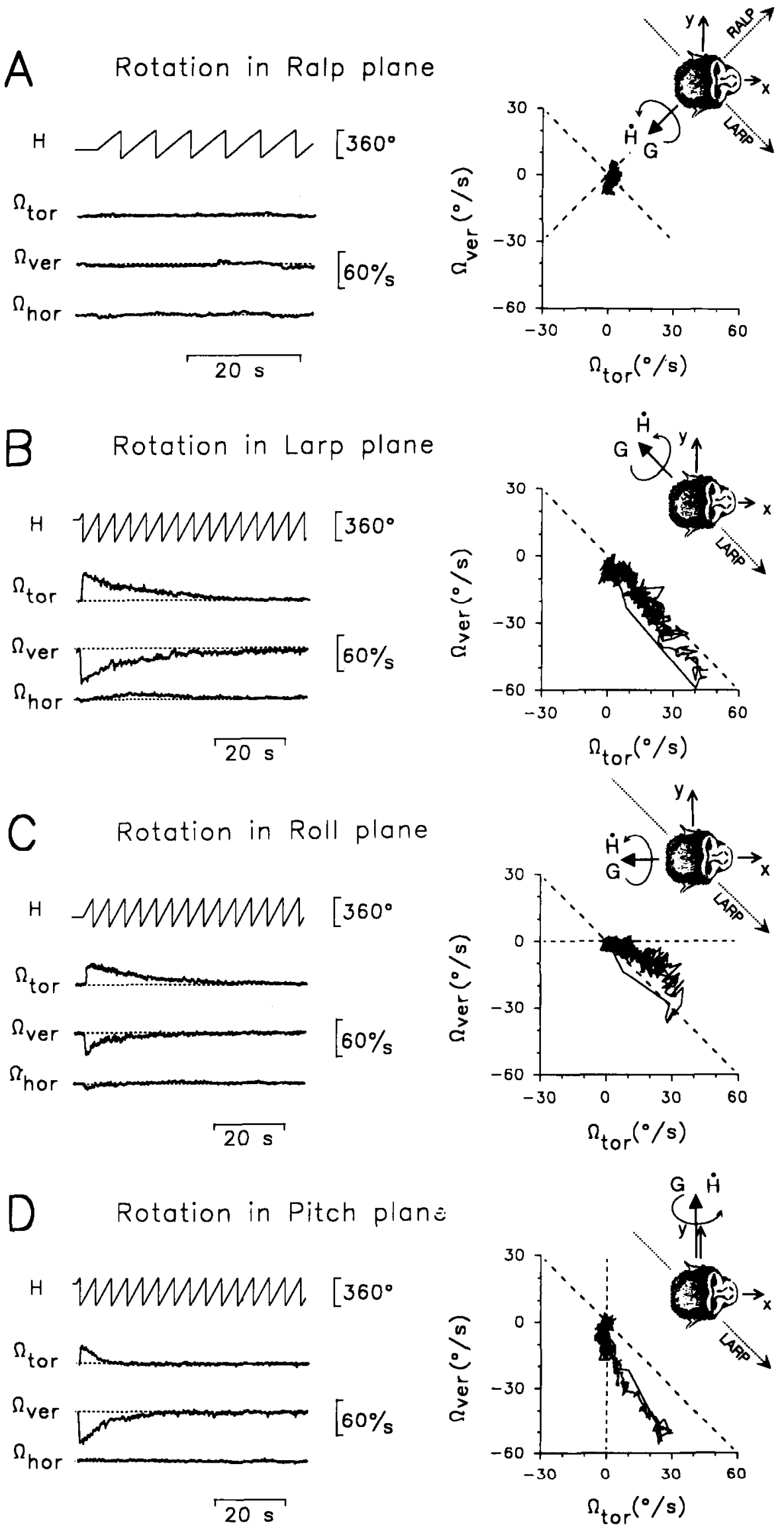


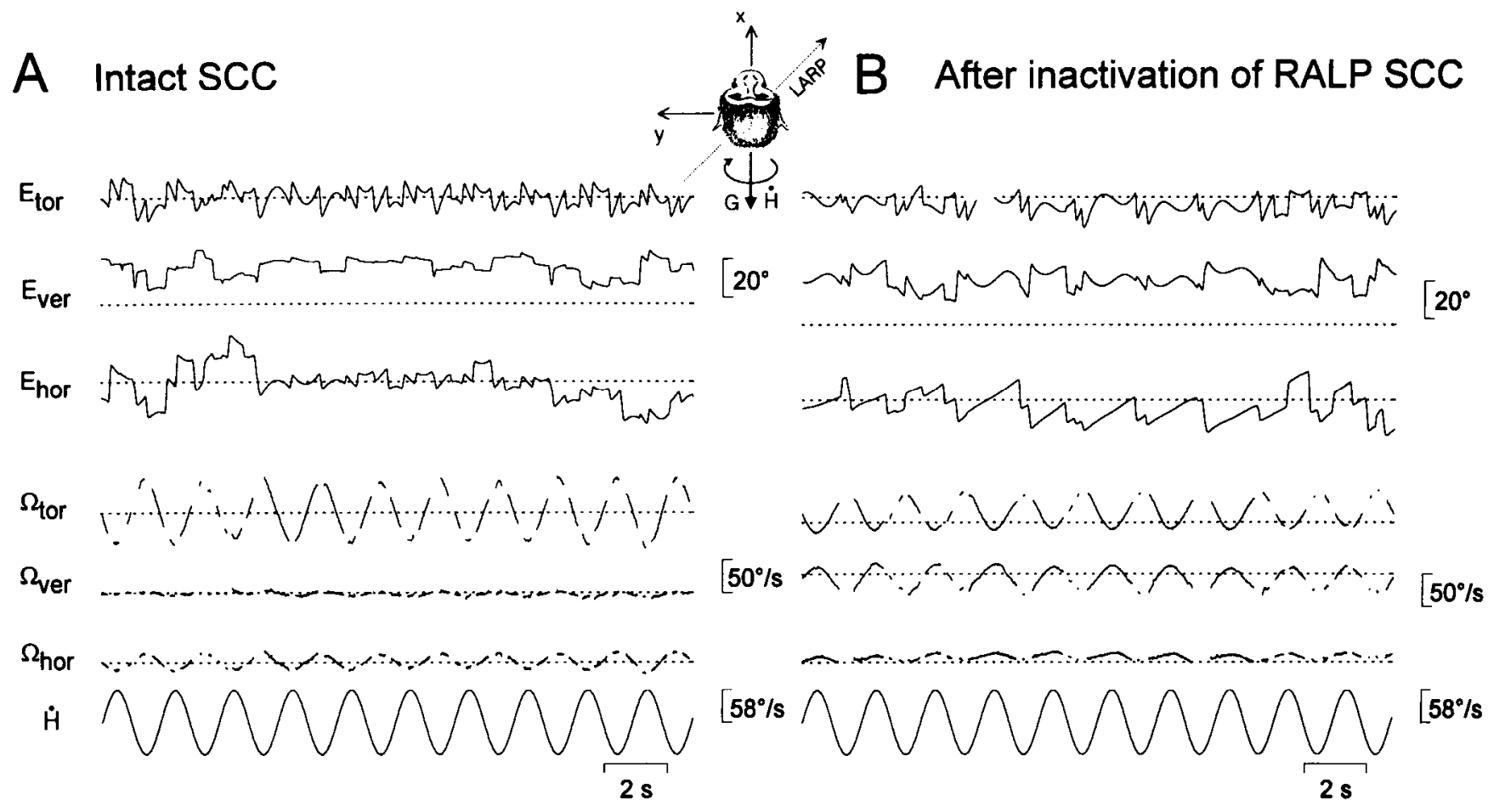

Figure 7. Torsional $\left(E_{\mathrm{tur}}, \Omega_{\mathrm{tor}}\right)$, vertical $\left(E_{\mathrm{ver}}, \Omega_{\mathrm{ver}}\right)$, and horizontal $\left(E_{\mathrm{hor}}, \Omega_{\mathrm{hor}}\right)$ eye position and slow phase eye velocity (fast phases removed) during $0.5 \mathrm{~Hz}$ oscillations in supine position. $A$. Responses in an animal with all semicircular canals (SCC) intact. $B$, Responses acutely after inactivation of the right anterior/left posterior (RALP) canals. Dotted lines mark zero eye position and velocity. $\mathbf{H}$, Head velocity vector (direction in head insets corresponds to the stimulus polarity that elicits positive torsional nystagmus).

component during rotation in ear-down position. These time constant differences resulted in a curved slow phase velocity trajectory. During rotation in supine position, slow phase velocity was initially closely aligned with the LARP axis, and then gradually deviated towards the $\mathrm{x}$-axis (Fig. $6 \mathrm{C}$ ). Similarly, during rotation in ear-down position, slow phase velocity was initially approximately parallel to the LARP axis and then deviated towards the $y$-axis (Fig. 6D). Thus, in both examples, the late VOR response was closer aligned with the earth-vertical direction than the initial response which was always along the LARP axis.

These differences in the dynamic behavior of torsional and vertical response components during rotation in supine and eardown positions became even more evident during sinusoidal stimulation. Torsional, vertical, and horizontal eye position and slow phase eye velocity components during $0.5 \mathrm{~Hz}$ oscillations in supine position in the same animal before and acutely after right anterior/left posterior semicircular canal inactivation are compared in Figure 7, $A$ and $B$, respectively. Earth-vertical axis rotation of an animal with intact semicircular canals in supine position elicits mainly a torsional nystagmus with negligible vertical components (Fig. 7A). After inactivation of the right anterior/left posterior canals, the same stimulus of $0.5 \mathrm{~Hz}$ oscillations elicits a combination of vertical and torsional cye movements (Fig. $7 B$ ). The ratio between vertical and torsional response amplitudes during oscillations in ear-down and supine positions was found to be a function of stimulus frequency. In fact, the following spatial response pattern emerged: At $0.5 \mathrm{~Hz}$, the torsional and vertical slow phase velocity components were approximately of equal amplitude in both ear-down and supine positions
(Figs. $7 B, 8$, top panels). ${ }^{2}$ At $0.01 \mathrm{~Hz}$, on the other hand, the vertical amplitude was greater than the torsional response amplitude during rotation in ear-down position. Similarly, the torsional response amplitude was greater than the vertical amplitude during rotation in supine position (Fig. 8, bottom). Gain and phase of torsional and vertical response components during sinusoidal oscillations at different frequencies in ear-down (pitch VOR) and supine (roll VOR) positions are illustrated in Figure 9. In the intact animal, rotation in ear-down position elicited mainly a vertical response, whereas rotation in supine position elicitcd mainly a torsional response (Fig. 9, open symbols). $\Lambda$ fter right anterior/left posterior canal inactivation, rotation in both ear-down and supine positions resulted in both vertical and torsional eye movements due to activation of the remaining intact vertical semicircular canal pair. At frequencies above approximately $0.1 \mathrm{~Hz}$, the amplitudes of both vertical and torsional response components remained constant, approximating 35-50\% of the response amplitude befure plugging (compare solid with open circles and squares in Fig. 9). During rotation in ear-down position at frequencies below $0.1 \mathrm{~Hz}$, torsional response amplitude decreased, whereas vertical response amplitude exhibited only a moderate change (Fig. 9, left). Similarly, during rotation in supine position at frequencies below $0.1 \mathrm{~Hz}$, vertical response

\footnotetext{
${ }^{2}$ The small horizontal response components elicited during sinusoidal oscillation at $0.5 \mathrm{~Hz}$ in supine and ear-down positions $\left(\Omega_{\text {hor }}\right.$ in Fig. 8 ) had a gain of approximately $10-20 \%$ of the vertical component during rotation in eardown positions before plugging. This horizontal component reflects a contribution from the lateral canals during pure pitch and roll rotations. In intact animals, no such horizontal component was present since it is canceled by signals from vertical canals. Notice also that no such horzontal component was present during oscillation at $0.01 \mathrm{~Hz}$, which reflects the fact that low frequency VOR responses are dominated by inertial vestibular signals.
} 

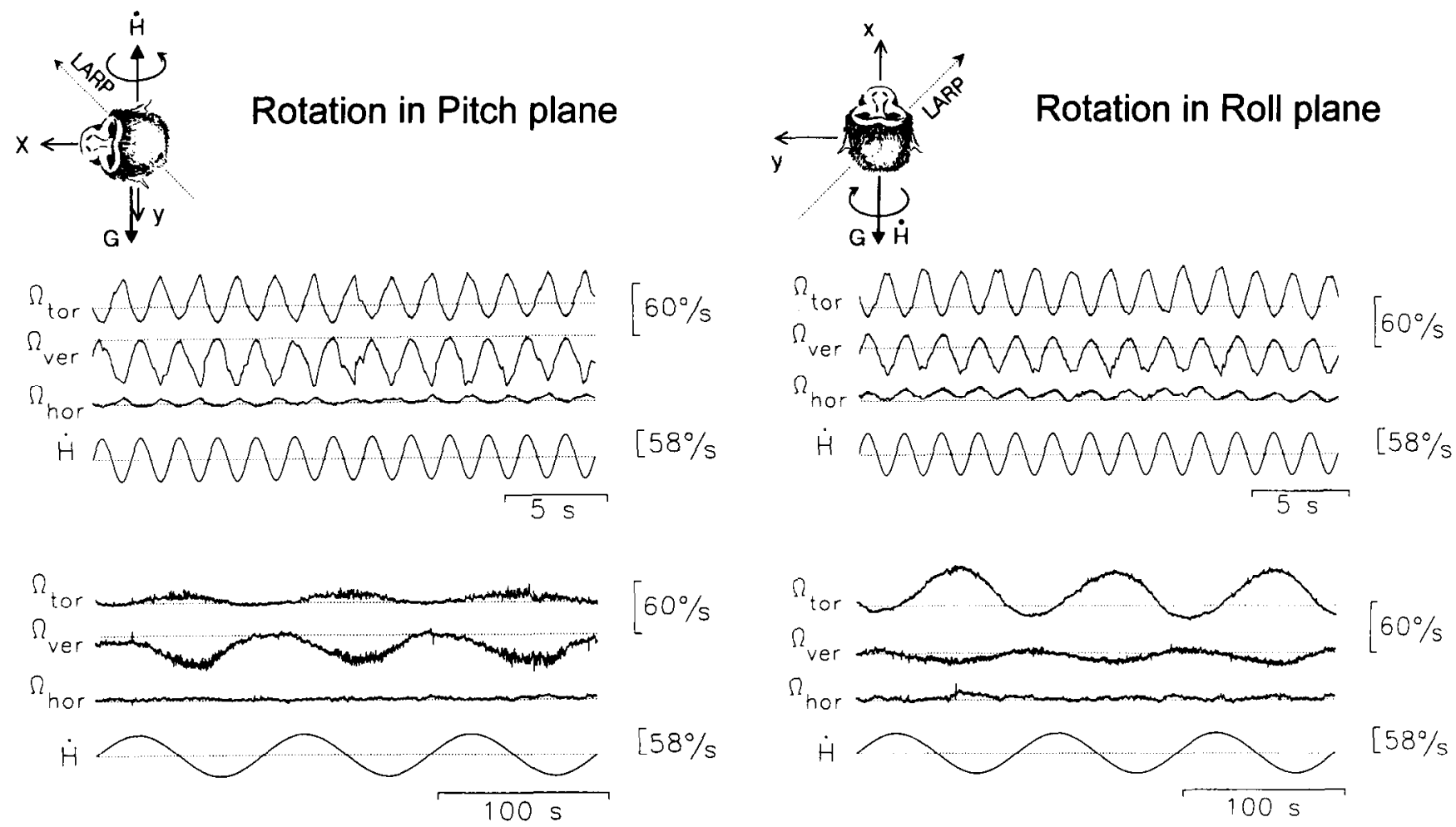

Figure 8. Low versus high frequency sinusoidal VOR in the pitch and roll planes. Left, Pitch VOR in left ear-down position. Right, Roll VOR in supine position. Torsional $\left(\Omega_{\mathrm{tor}}\right)$, vertical $\left(\Omega_{\mathrm{ver}}\right)$, and horizontal $\left(\Omega_{\mathrm{hwr}}\right)$ slow phase eye velocity responses elicited during sinusoidal oscillation at $0.5 \mathrm{~Hz}(t g p)$ and $0.01 \mathrm{~Hz}$ (bottom) acutely after inactivation of the right anterior/left posterior semicircular canals. Dotted lines mark zero eye velocity. $\mathbf{H}$, Head velocity vector: the direction in head insets corresponds to the stimulus polarity that elicits positive torsional (right) or vertical (left) nystagmus.

Figure 9. Dynamics of pitch and roll VOR acutely after inactivation of the right anterior/left posterior semicircular canals. Response gain and phase of the torsional (squares) and vertical (circles) response components are com pared with control data before semicircular canal inactivation (open symbols). The torsional and vertical response components during pitch and roll VOR, respectively, are very small in the intact animal (gains $<0.08$ not illustrated). Gain of each response component has been estimated as the ratio of response amplitude over peak head velocity.
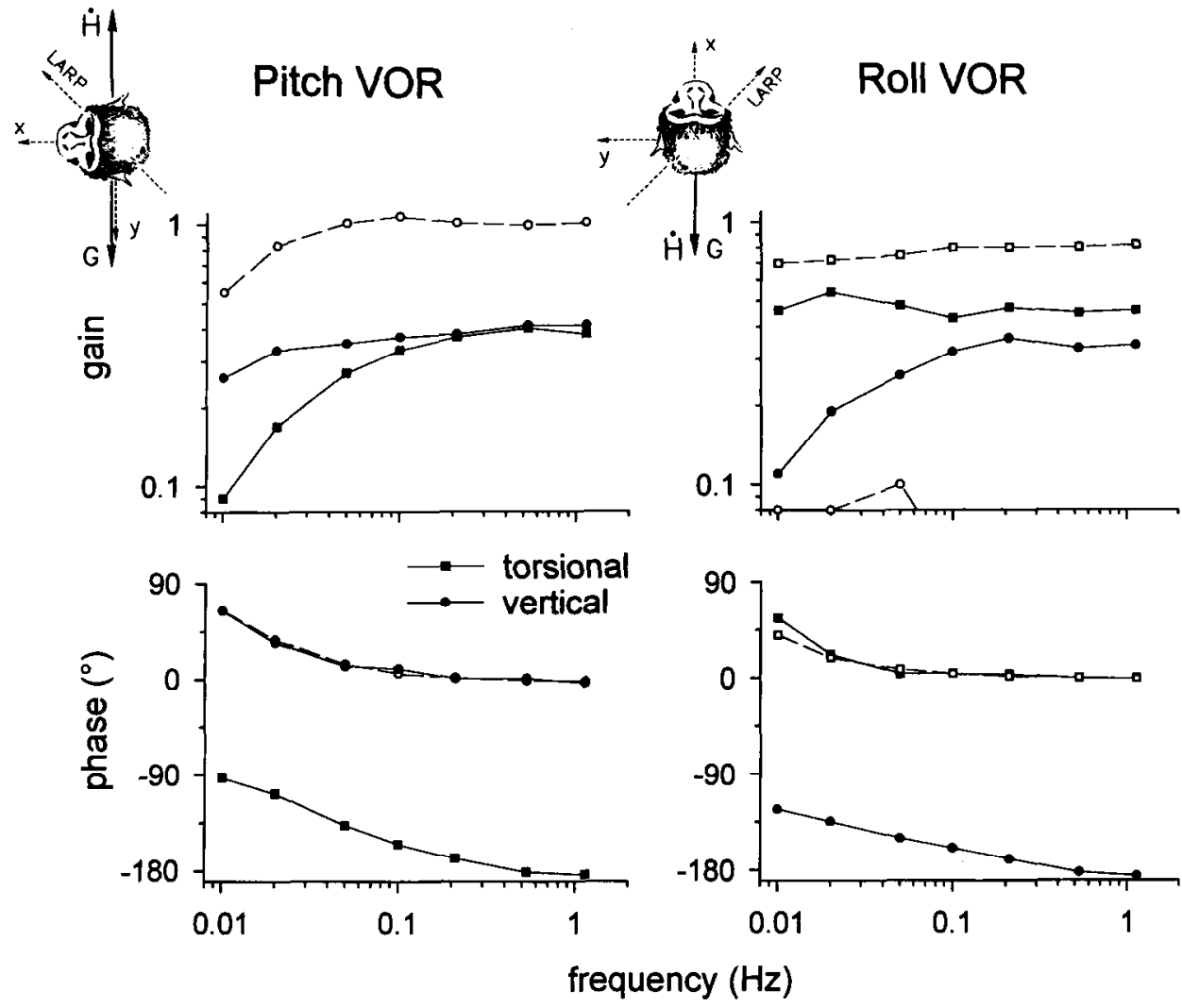


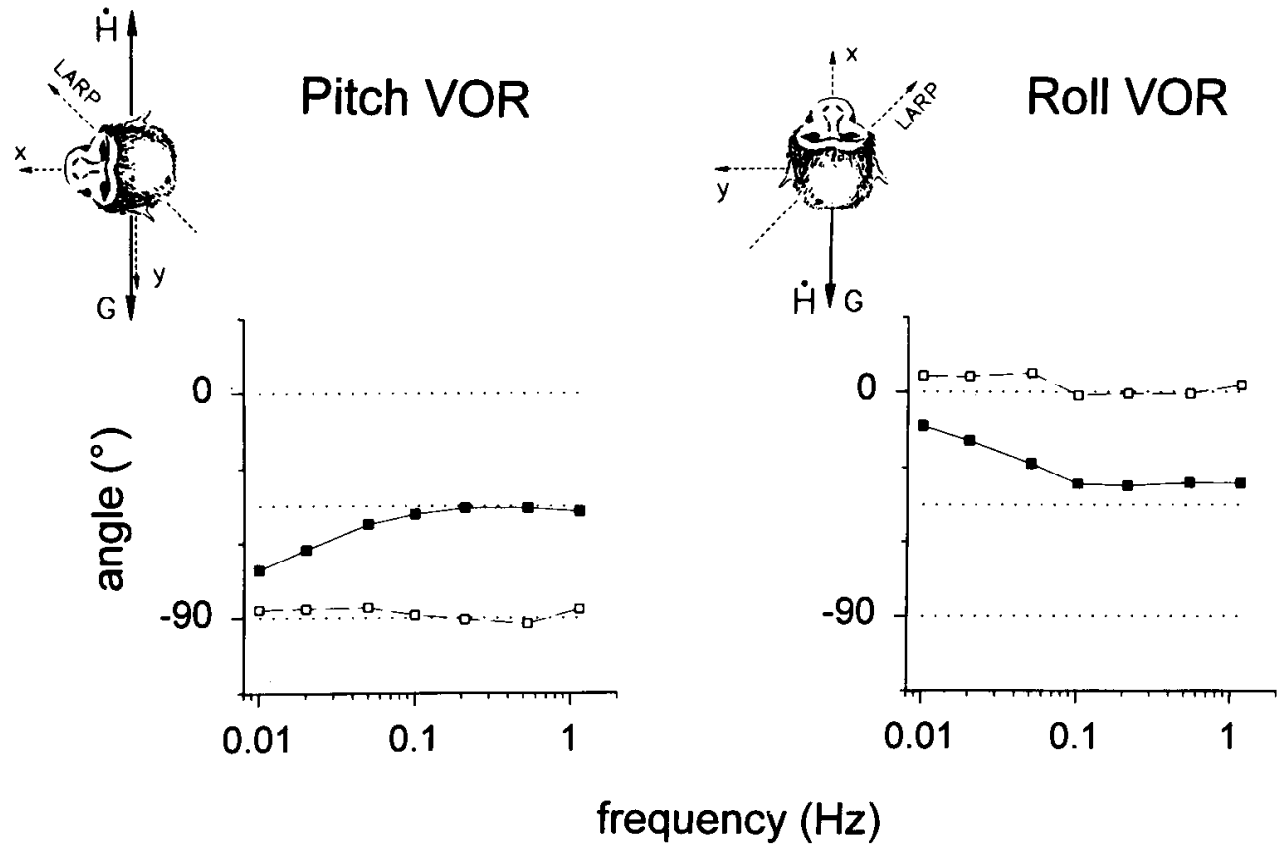

Figure 10. Vector orientation of slow phase eye velocity during pitch and roll VOR before and acutely after inactivation of the right anterior/left posterior canals (open versus solid squares) (same data as in Fig. 9). Pure vertical and torsional slow phase eye velocity tended to align with the dashed lines at $-90^{\circ}$ and $0^{\circ}$, respectively. A slow phase eye velocity response along the LARP axis would be oriented at $45^{\circ}$. amplitude decreased, while torsional response amplitude remained relatively constant (or actually increased) at low frequencies (Fig. 9, right). Interestingly, these large relative changes in gain were accompanied by no or only minor differences in phase.

The spatial orientation of VOR slow phase velocity in the yaw plane was estimated by computing the inverse tangent of the amplitude ratio of the vertical and torsional response components (response amplitudes were considered positive when phase was between $-90^{\circ}$ and $90^{\circ}$ and negative otherwise). At mid and high frequencies slow phase velocity was parallel to the LARP axis (i.e., at a $-45^{\circ}$ orientation) independently of whether rotation was about the pitch or roll axis (Fig. 10). At frequencies below $0.1 \mathrm{~Hz}$, the orientation of VOR slow phase velocity changed gradually as frequency decreased. During rotation in ear-down position, the orientation of VOR approached $90^{\circ}$ at $0.01 \mathrm{~Hz}$, that is, responses were nearly vertical as before inactivation of the RAI.P canals (compare solid and open squares in Fig. 10, left). Similarly, during rotation in supine position, the orientation of VOR slow phase velocity approached 0 at 0.01 $\mathrm{Hz}$, that is, responses became nearly torsional as in intact animals (compare solid and open squares in Fig. 10, right).

Similar results were obtained in the animal with three inactivated vertical canals (Fig. 11). Since only one vertical canal remained intact (left posterior canal), the amplitudes of both vertical and torsional response components were smaller than those observed in the animal with inactivated right anterior/left posterior canals. Nevertheless, the spatial response characteristics exhibited a similar dichotomy in the low versus mid and high frequency range. During mid and high frequency head rotation in ear-down and supine positions, the relative amplitudes of vertical and torsional slow-phase velocity were roughly equal, resulting in a VOR aligned with the RALP axis (i.e., at a $45^{\circ}$ orientation; see inset in Fig. 11). As frequency decreased below $0.1 \mathrm{~Hz}$, the amplitude of the torsional slow-phase velocity decreased sharply during rotation in ear-down position, while it remained relatively constant during rotation in supine position (solid squares in Fig. 11, top). Similarly, the amplitude of the vertical slow-phase velocity remained relatively constant during rotation in ear-down position but decreased sharply during rotation in supine position (solid circles in Fig. 11, top). As a result, the orientation of slow phase eye velocity approached $90^{\circ}$ during rotation in ear-down position and $0^{\circ}$ during rotation in supine position (solid diamonds in Fig. 11, bottom).

These results demonstrate that the low frequency VOR does not simply reflect an angular velocity signal in proportion to the activated semicircular canals but rather a modified head angular velocity signal after spatial transformation based on head position information. In the following, we present a quantitative analysis of these responses focusing on the response patterns found after inactivation of the right anterior/left posterior canals and after bilateral inactivation of the lateral canals. Since responses obtained from the animal with all but one vertical canal inactivated were similar, they will not be presented in any further detail. Experimental responses will be compared with model simulations based on two different representations of vestibular signals: a head-fixed and an inertial, space (or gravity)-fixed representation of head velocity signals.

\section{Head-fixed and space-referenced inertial vestibular signals}

\section{VOR spatial organization in the yaw plane}

In these experiments, the animal's head orientation was systematically varied relative to gravity such that the vector of imposed head velocity $(\dot{\mathbf{H}})$ changed its orientation in the yaw plane in steps of $15^{\circ}$ or $30^{\circ}$ (Fig. 12). Accordingly, an angular orientation of $\alpha=0^{\circ}$ or $180^{\circ}$ corresponded to rotation about an earth-vertical axis in prone or supine position, respectively, and an angle of $\alpha= \pm 90^{\circ}$ corresponded to a rotation in ear-down positions. After inactivation of the right anterior/left posterior vertical semicircular canals, only the left anterior/right posterior canals could detect a head angular velocity component in the yaw plane. If the VOR were only a function of semicircular canal afferent input, the spatial orientation of VOR slow phase velocity after right anterior/left posterior canal inactivation would be independent of the angle $\alpha$. Even though the relative amplitudes 

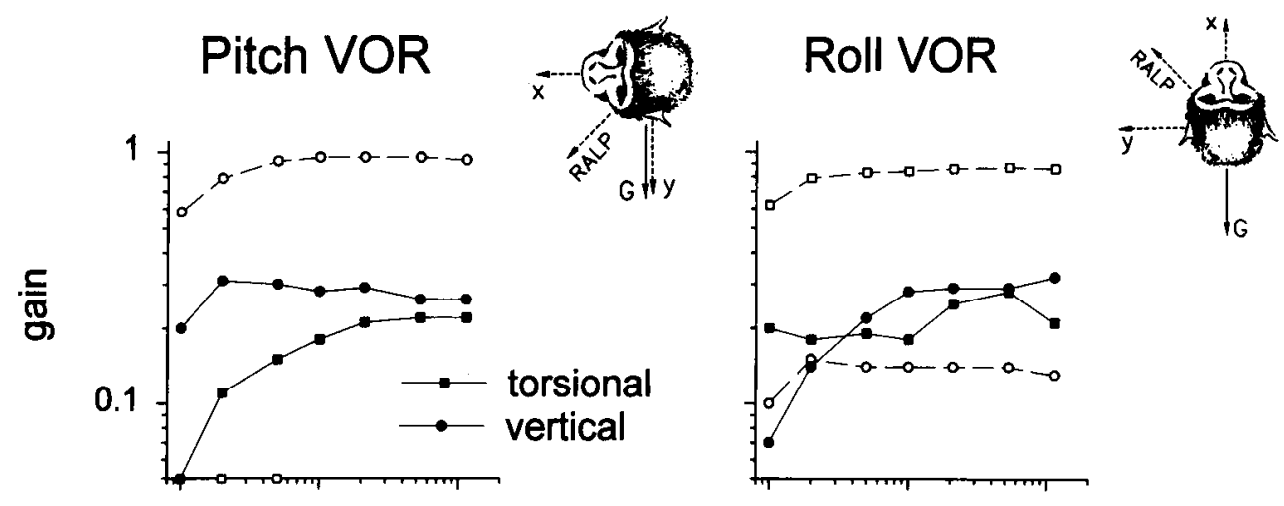

Figure 11. Gain and spatial orientation of the pitch and roll VOR acutely after inactivation of the lateral, right anterior, left anterior and right posterior semicircular canals. Circles, Vertical response components; squares, torsional response components; diamonds, orientation of slow phase eye velocity vector. Open symbols, intact animal; solid symbols, data after semicircular canal inactivation.

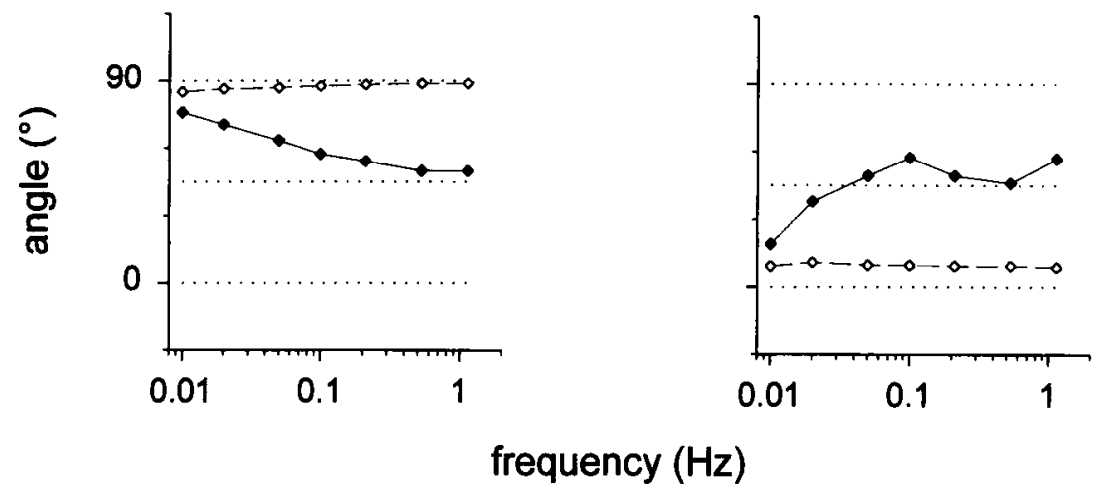

of vertical and torsional velocity components would vary as the direction of imposed head velocity changed in the yaw plane, their amplitude ratio should always be such that the angular orientation of the slow phase cyc velocity vector remains close to $-45^{\circ}$, that is, parallel to the LARP axis (Fig. 12, top). If the VOR, on the other hand, were also dependent on space-referenced inertial vestibulo-ocular signals, one might expect that semicircular canal signals undergo a spatial transformation such that the vector of eye velocity and the direction of gravity (and the stimulus) align (angle $\alpha$ in Fig. 12, top).

The experimental results showed that VOR slow phase velocity at 0.2 and $1 \mathrm{~Hz}$ remained at a constant orientation of approximately $-45^{\circ}$ independently of the angular orientation of head velocity in the yaw plane, as expected for solely semicircular canal-based vestibulo-ocular signals. In contrast, slow phase eye velocity at $0.01 \mathrm{~Hz}$ was aligned with the direction of gravity, similarly to responses prior to semicircular canal inactivation (Fig. 12, solid vs open circles). A similar frequencyspecific response pattern was also apparent when plotting the amplitude of the elicited vertical and torsional response components as a function of stimulus angle $\alpha$ (Fig. 13). At $0.2 \mathrm{~Hz}$ and $1 \mathrm{~Hz}$, vertical and torsional response amplitude varied as a cosine function of stimulus angle $\alpha$, and reached peak values simultaneously at $-45^{\circ}$ and $135^{\circ}$ when the intact LARP semicircular canals were maximally activated. Orthogonal to this plane, both vertical and torsional response components, as well as the resultant slow phase velocity were zero (Fig. 13, lower two panels). At $0.01 \mathrm{~Hz}$, on the other hand, vertical and torsional response amplitudes no longer exhibited a cosine tuning behavior. The torsional and vertical response profiles were symmetrically shifted relative to each other such that vertical response peaked at approximately $-60^{\circ}$ and $120^{\circ}$, whereas torsional response peaked at approximately $-30^{\circ}$ and $150^{\circ}$. The resultant slow phase velocity peaked at an angle of approximately $-45^{\circ}$ and $135^{\circ}$ and became zero at $45^{\circ}$ and $-135^{\circ}$ consistent with a single functional vertical canal pair (Fig. 13, top panel).

The functional significance of these experimental findings can best be illustrated by the following geometrical considerations (Fig. 14).

Head-fixed coding of head angular velocity. In this case, the internally coded head angular velocity $\mathbf{v}$ is exclusively a function of semicircular canal inputs and thus proportional to the projection of the vector of head angular velocity $\dot{\mathbf{H}}$ onto the LARP axis. Thus, we find for the vertical $\left(v_{y}\right)$ and torsional $\left(v_{x}\right)$ VOR slow phase eye velocity components (Fig. 14A, left):

$$
\begin{aligned}
& v_{x} \approx \cos \left(\alpha+45^{\circ}\right) \cos 45^{\circ}, \\
& v_{y} \approx \cos \left(\alpha+45^{\circ}\right) \sin 45^{\circ} .
\end{aligned}
$$

These equations predict that both the vertical and torsional response components should vary as the cosine of angle $\alpha$, and peak for $\alpha=-45^{\circ}$ (or $135^{\circ}$ ) when the vector of head velocity $\dot{\mathbf{H}}$ is parallel to the LARP sensitivity vector (Fig. 14B, top). At $\alpha=45^{\circ}$ (or $-135^{\circ}$ ), both vertical and torsional response components (solid and dashed lines coincide in Fig. 14B, top) as well as the resultant slow phase velocity (dotted lines in Fig. $14 B$, top) would be zero.

Space (gravity)-fixed coding of head angular velocity. In this case, vestibulo-ocular signals are assumed to be space (gravity)fixed based on a spatial transformation of afferent signals. As a consequence, vertical and torsional response amplitudes exhibit a more complex dependency on angle $\alpha$. As in the previous case, the head angular velocity coded by semicircular canal afferents is proportional to the projection of head angular velocity $\dot{\mathbf{H}}$ onto the LARP sensitivity vector. In order to represent head velocity in space, however, these signals need to be spatially transformed, for example, by projecting the component of head velocity 

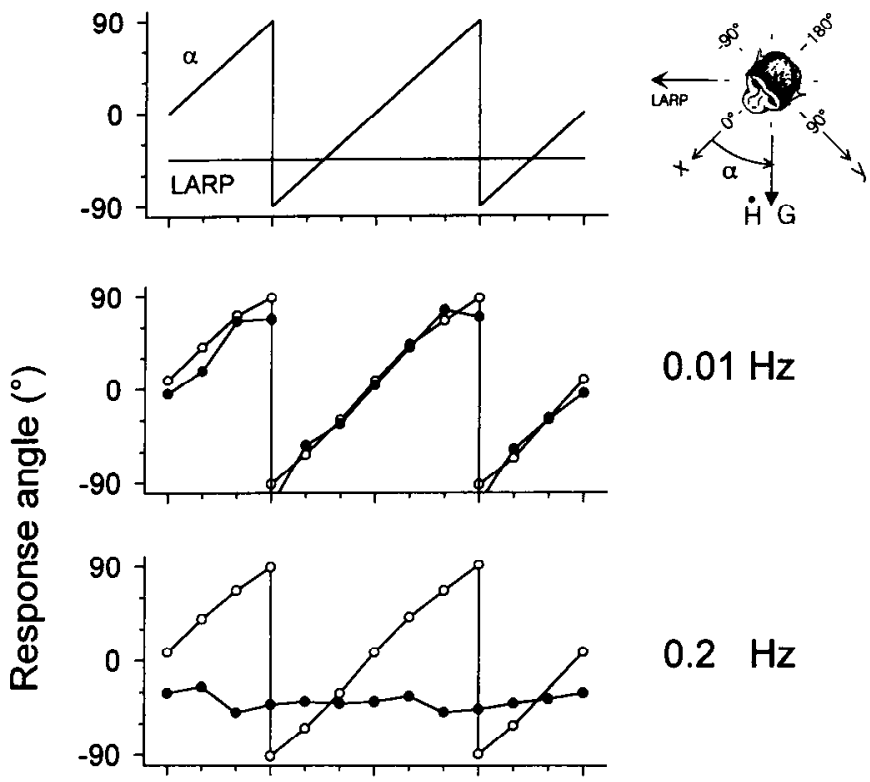

\section{$0.01 \mathrm{~Hz}$}

\section{$0.2 \mathrm{~Hz}$}

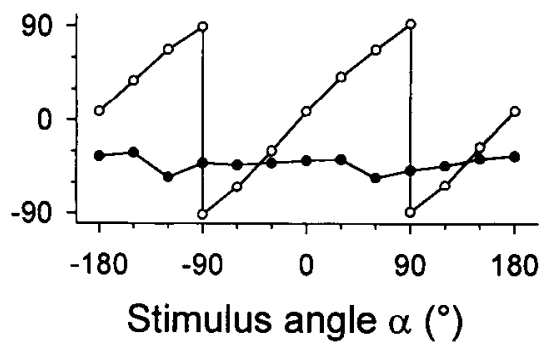

$1 \mathrm{~Hz}$

Figure 12. Spatial orientation of VOR slow phase eye velocity as a function of the orientation of the stimulus (and gravity) acutely after inactivation of the right anterior/left posterior canals. The orientation of the vector of head velocity $\mathbf{H}$ (and $\mathbf{G}$ ) are defined by the polar angle $\alpha$ relative to the positive $\mathrm{x}$-axis (see inset). Top panel, if VOR remains spatially aligned with the stimulus $\mathbf{H}$ (and $\mathbf{G}$ ), response angle and stimulus angle $\alpha$ would be identical (i.e., response angle would follow a sawtooth line). If VOR response is independent of stimulus orientation and always parallel to the LARP axis, response angle would be constant (i.e., along line at $-45^{\circ}$ ). Lower three panels, experimental data at 0.01 , 0.2 , and $1 \mathrm{~Hz}$. Open symbols, intact animal. Solid symbols, data after semicircular canal inactivation.

picked up by the intact LARP canals onto the direction of gravity (Fig. 14A, right). As a result of such transformation, the angular head velocity vector $\dot{\mathbf{H}}$ would be expressed in the VOR as a slow phase vector $\omega$ coaxial with gravity having components $\omega_{x}$ and $\omega_{y}$ proportional to

$$
\begin{aligned}
& \omega_{x} \approx\left(\cos \left(\alpha+45^{\circ}\right)\right)^{2} \cos \alpha, \\
& \omega_{y} \approx\left(\cos \left(\alpha+45^{\circ}\right)\right)^{2} \sin \alpha .
\end{aligned}
$$

As apparent from the simulations of the above equations, vertical and torsional response components no longer follow a rectified cosine profile as a function of angle $\alpha$ (Fig. 14B, bottom). In addition, the two components are phase-shitted by $30^{\circ}$ relative to each other (vertical response: solid line; torsional response: dashed line in Fig. $14 B$, bottom), cven though the resultant slow phase velocity still peaks at an angle of $-45^{\circ}$ and $135^{\circ}$ and becomes zero at an angle of $45^{\circ}$ or $-135^{\circ}$.

Other than some asymmetry in response amplitude in supine and prone positions, these simulations closely describe the experimental results. Oscillation at $0.2 \mathrm{~Hz}$ and $1 \mathrm{~Hz}$ elicited vertical and torsional response components consistent with head-
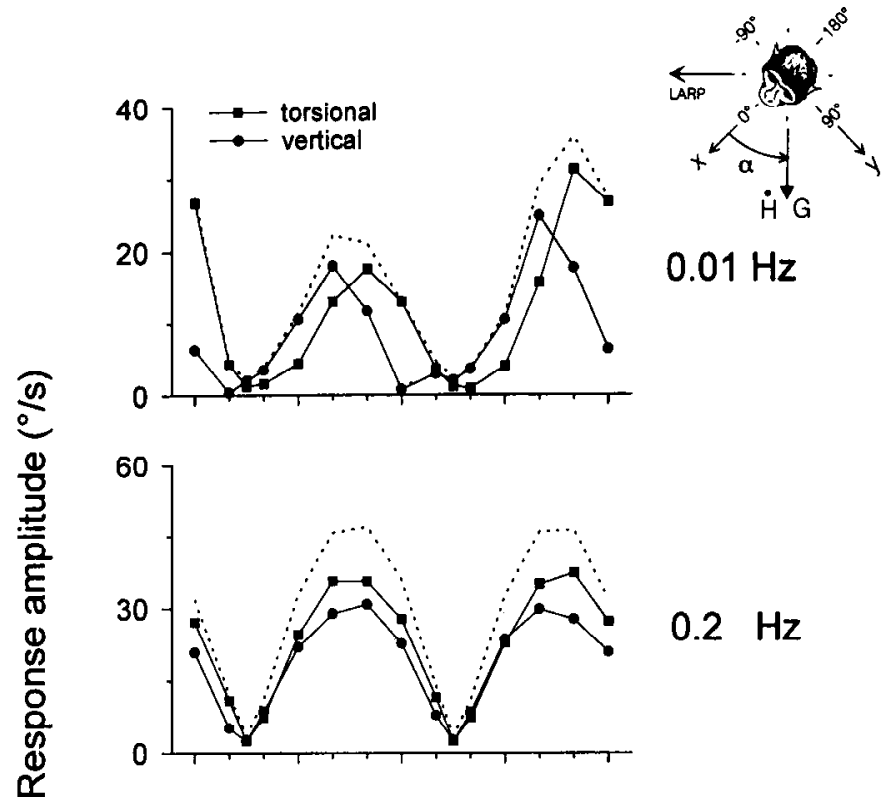

$0.01 \mathrm{~Hz}$

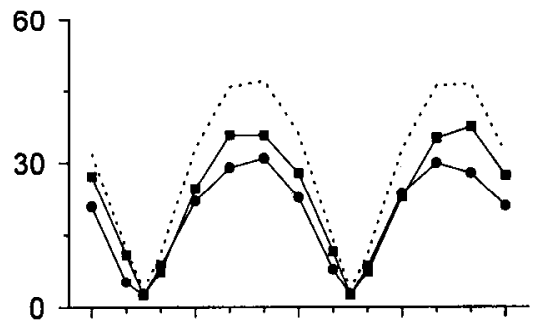

$0.2 \mathrm{~Hz}$

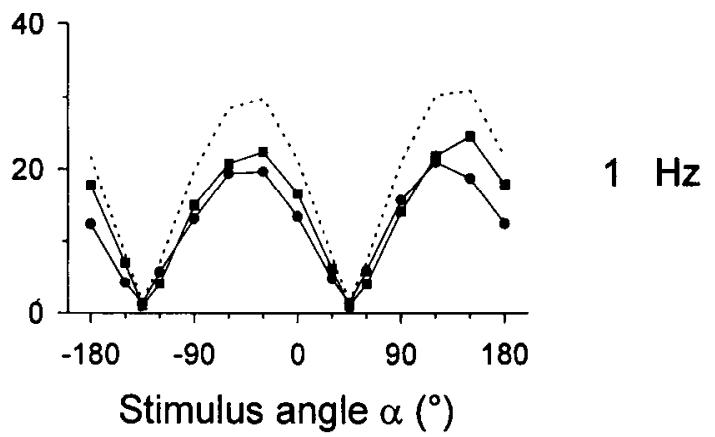

Figure 13. Torsional and vertical response components as a function of stimulus orientation in the yaw plane (squares and circles, respectively). Data obtained at three different oscillation frequencies are plotted versus stimulus angle $\alpha$ (see inset): $0.01 \mathrm{~Hz}$ (top), $0.2 \mathrm{~Hz}$ (middle), and $1 \mathrm{~Hz}$ (bottom). Dotted lines, resultant response in the yaw plane. Sante dala as in Figure 12.

fixed coding of angular velocity $\mathbf{v}$ (compare Fig. 13, middle and bottom, and Fig. 14B, top). In contrast, vertical and torsional response amplitude at $0.01 \mathrm{~Hz}$ followed the expected response profile of a space-fixed inertially coded angular velocity $\omega$ (Fig. 13, top, and Fig. 14, bottom).

So far we have examined the spatial organization of the VOR for head velocity vectors in the yaw plane. These responses reflect the spatial organization of vertical canal-born head velocity signals. The following paragraphs will examine the spatial organization of the VOR in vertical head planes. Depending on the plane tested, these data will reflect the spatial organization of either lateral canal-born head velocity signals alone or lateral and vertical canal-born head velocity signals together. For simplicity, only slow phase velocity amplitudes will be presented and compared with model predictions. If amplitudes of each VOR component are adequately predicted by the model, so would also the spatial orientation of VOR slow phase velocity.

\section{VOR spatial organization in vertical head planes}

After right anterior/left posterior semicircular canal inactivation. To examine the spatial organization of lateral canal signals alone, the monkey with inactivated right anterior/left posterior canals was systematically tested at different positions relative to 

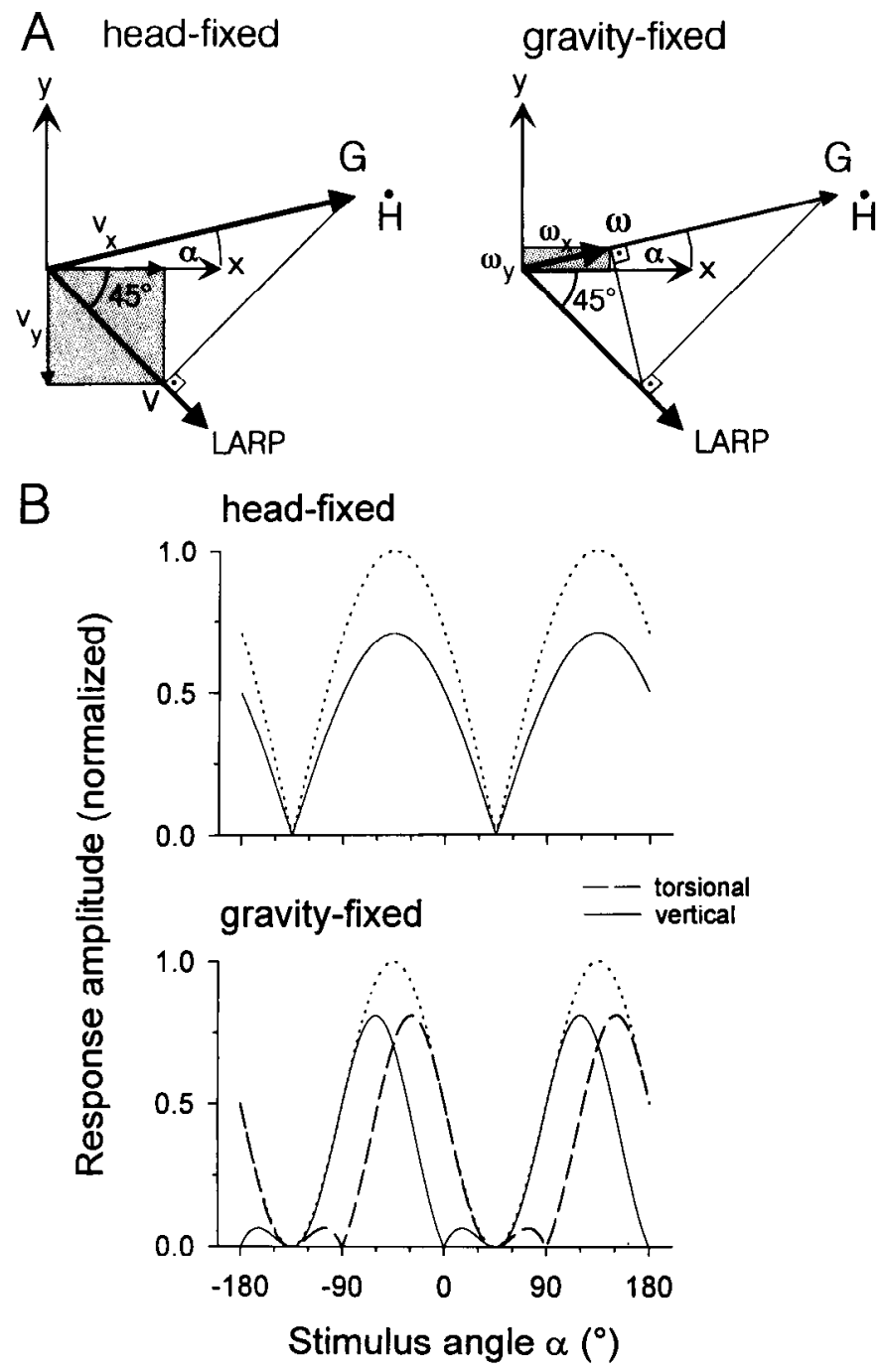

Figure 14. Predicted torsional and vertical response components for head-fixed and gravity-fixed central representation of vertical semicircular canal signals. $A$, Geometric relationship between the vector of head angular velocity and a head-fixed $\mathbf{v}$ or a space-fixed central response vector $\boldsymbol{\omega}$ (diagonals of the shaded rectangular areas). B Simulations of vertical (solid lines), torsional (dashed lines), and resultant response (dotted lines) based on the head-fixed (top) and the spacefixed inertial model (bottom). Note that the vertical and torsional components coincide in the head-fixed model.

gravity while he was tilted systematically in the head LARP plane (Fig. 15). A tilt angle of $0^{\circ}$ corresponded to upright position, whereas angles of $90^{\circ}$ corresponded to nose-up/right eardown and nose-down/left ear-down positions. Under the assumption of canal orthogonality (see Materials and Methods), only the intact lateral semicircular canals were activated during earth-vertical axis rotation in any of these head positions (except for $\pm 90^{\circ}$ positions where rotation was in the plane of the inactivated canals). If lateral semicircular canal signals were processed independently of head orientation relative to gravity, only horizontal eye movements would be generated for any of these stimulus orientations. As simulations demonstrate (Fig. 15A, top), horizontal slow phase velocity should simply vary as a cosine function of stimulus orientation and the vertical/torsional components should be zero for any head position. Alternatively, if vestibulo-ocular signals originating from lateral semicircular canal activity were processed by an inertial vestibular system
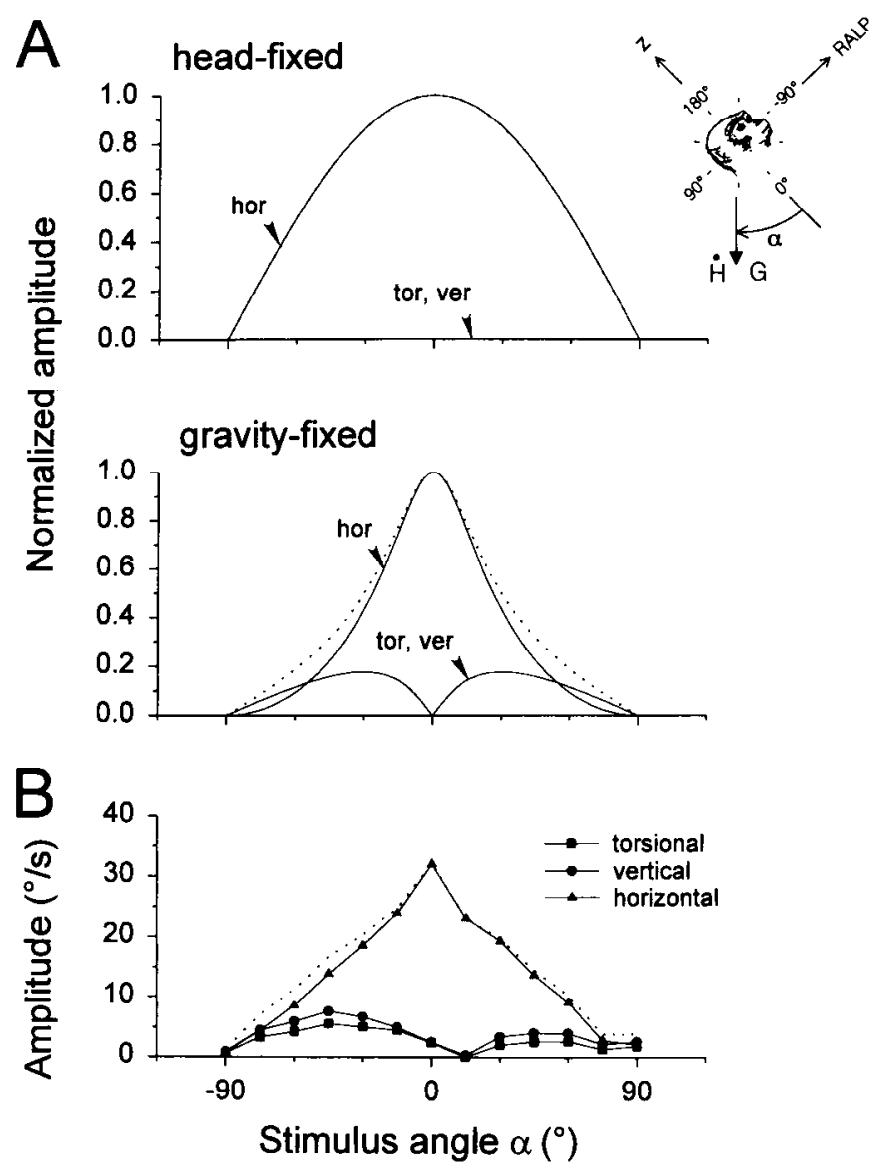

Figure 15. Low frequency VOR organization in the LARP plane (right anterior/left posterior canals inactivated). $A$, Simulations of a head fixed (top) and space fixed (bottom) representation of vestibuloocular signals. The space-fixed inertial model was based on a rotation and contraction of lateral canal activity. Solid lines, horizontal, vertical, and torsional components as indicaled by arrowheads. Dotted lines, resultant response amplitude. B, Horizontal (triangles), vertical (circles), torsional (squares), and resultant (dotted lines) slow phase eye velocity as a function of head orientation in the LARP plane (angle $\alpha$; see inset). Data recorded 7 months after semicircular canal inactivation $(0.01 \mathrm{~Hz})$.

that transforms afferent signals according to head orientation in space, horizontal slow phase velocity should exhibit more narrowly tuned response characteristics as a function of stimulus angle (Fig. 15A, bottom). Moreover, torsional and vertical response components should be generated in head positions tilted away from upright. The spatial transformation adopted in these simulations corresponded to a combination of rotation and amplitude contraction which has been previously used to explain the spatial reorientation of horizontal postrotatory responses af ter fast head tilts (Angelaki and Hess, 1994, 1995; see Materials and Methods).

The mid and high frequency VOR responses obtained in the animal with inactivated right anterior/left posterior canals were, in fact, consistent with the hypothesis of a head-fixed representation of angular head velocity (data not shown). However, low frequency VOR responses were consistent with the hypothesis that head angular velocity signals arising from the lateral canals were transformed relative to space (Fig. 15B). Some differences between experimental data and simulations were present. The model simulation predicted a sharper decrease of the horizontal response component as angle $\alpha$ increased. In contrast, experi- 
mental data exhibited a relatively sharp decrease in horizontal amplitude only at small angles around $0^{\circ}$ and a less sharp decrease at larger angles, suggesting that there was relatively strong amplitude contraction at small angles and much less at large angles. Thus, it seems that the contraction factor $c(\alpha)$ used in this model (Eq. 3; see Materials and Methods) exhibits a more complex profile as a function of angle $\alpha$. Since the exact profile of the contraction $c(\alpha)$ does not affect our main conclusions, Equation 3 was used throughout the present simulations.

Simulations of the spatial organization of the VOR in the roll and pitch planes are somewhat more involved. As the stimulus angle varies in the roll or pitch plane, both the intact lateral canals and the intact left anterior/right posterior canals are stimulated. If in this case semicircular canal signals were spatially reorganized based on head orientation relative to gravity, lateral canal activity would be expected to be transformed by rotation and amplitude contraction, whereas vertical canal activity would be transformed by projection. In the following model simulations, we have taken into account these two transformation schemes. In a first condition, ${ }^{3}$ we assumed that only lateral canal activity was spatially transformed whereas processing of vertical canal activity remained head-fixed, as proposed by Raphan and Sturm, 1991 (also Raphan et al., 1994). In the second condition, we assumed that both lateral and vertical semicircular canal activity were spatially transformed (Angelaki and Hess, 1994, 1995). Comparison of the two model simulations for the pitch plane shows differences mainly with regard to the amplitude of the vertical component (Fig. 16A). If only lateral canal activity was kept invariant relative to space, both vertical and torsional response components should be elicited as the head was tilted away from upright towards prone and supine positions (Fig. $16 A$, top). If all semicircular canal signals were transformed, only a torsional but no vertical response component would be present for any angle of stimulation in the pitch head plane (Fig. $16 \mathrm{~A}$, bottom). Our experimental results strongly support the latter hypothesis, namely a spatial transformation of all components of low frequency vestibulo-ocular signals. In support of this notion, there was a torsional but no consistent vertical response component that gradually increased as the head was tilted away from upright (Fig. 16B). As found above, the tuning curve of the horizontal component differed somewhat from the simulated profile, suggesting again a more complex amplitude contraction factor $c(\alpha)$. Similar results were obtained in the roll plane.

After lateral semicircular canal inactivation. The spatial organization of vertical semicircular canal signals was also investigated after bilateral inactivation of the lateral canals. A spatial transformation of vertical canal signals as a function of pitch position, for example, would follow the same projection principles as described above for the yaw plane. Thus, a head velocity vector $\dot{H}$ forming an angle $\alpha$ with the $\mathrm{z}$-axis would be picked up by the intact vertical canals and transformed further by projection onto the direction of gravity. Based on simple trigonometric calculations similar to those schematically shown in Figure 14, the angular head velocity vector $\dot{\mathbf{H}}$ would be transformed into a VOR slow phase vector $\omega$ parallel to gravity with components $\omega_{x}$ and $\omega_{y}$ proportional to

$$
\omega_{x}-\left(\cos \left(\alpha-90^{\circ}\right)\right)^{2} \cos \alpha,
$$

\footnotetext{
${ }^{3} \mathrm{~A}$ head-fixed $\mathrm{VOR}$ has not been simulated under these conditions because simulated results would be similar to those of Figure 15 with non-zero vertical and torsional components.
}
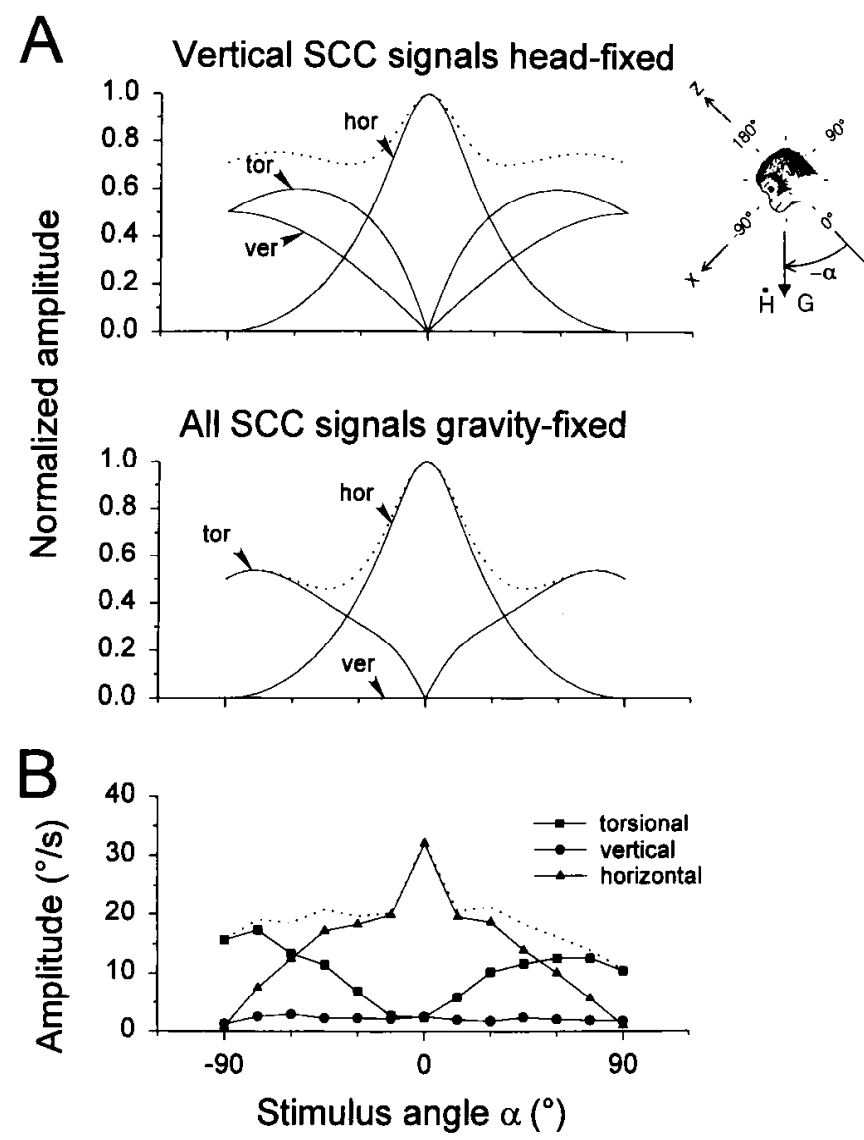

Figure 16. Low frequency VOR organization in the pitch plane (right anterior/left posterior canals inactivated). $A$, Simulations of two models, one in which only the lateral semicircular canal signals are spatially transformed while vertical semicircular canal $(S C C)$ signals remain head-fixed $(t o p)$ and another where all semicircular canal signals are transformed to relative space (bottom). Solid lines, horizontal, vertical, and torsional components as indicated by arrowheads. Dotted lines, resultant response amplitude. $B$, Horizontal (triangles), vertical (circles), torsional (squares), and resultant (dotted lines) response amplitudes as a function of stimulus orientation in the pitch plane (angle $\alpha$; see inset). Data recorded 7 months after semicircular canal inactivation $(0.01 \mathrm{~Hz})$.

$$
\omega_{y}-\left(\cos \left(\alpha-90^{\circ}\right)\right)^{2} \sin \alpha .
$$

Simulations of these equations show that the torsional response component of the transformed velocity signal no longer follows a rectified cosine function as would be the case for headfixed VOR signals (Fig. 17A, solid vs dashed lines). In addition, a horizontal component is predicted, which would be absent if vertical canal signals were processed independent of head position relative to gravity. Again, our results were consistent with head-fixed processing at mid and high frequency rotation (data not shown) and space (gravity)-fixed processing at low frequencies (Fig. 17B). In both animals a horizontal response component was generated during sinusoidal oscillation at $0.01 \mathrm{~Hz}$ when they were tilted towards supine. There was a pronounced asymmetry of responses in supine versus prone positions. This asymmetry was present acutely after lateral canal plugging and did not change thereafter. Notice that not only the horizontal but also the torsional response component was asymmetrically modulated as a function of head tilt. For example, even though the torsional response component peaked at an angle corresponding approximately to supine position, its amplitude continued to in- 

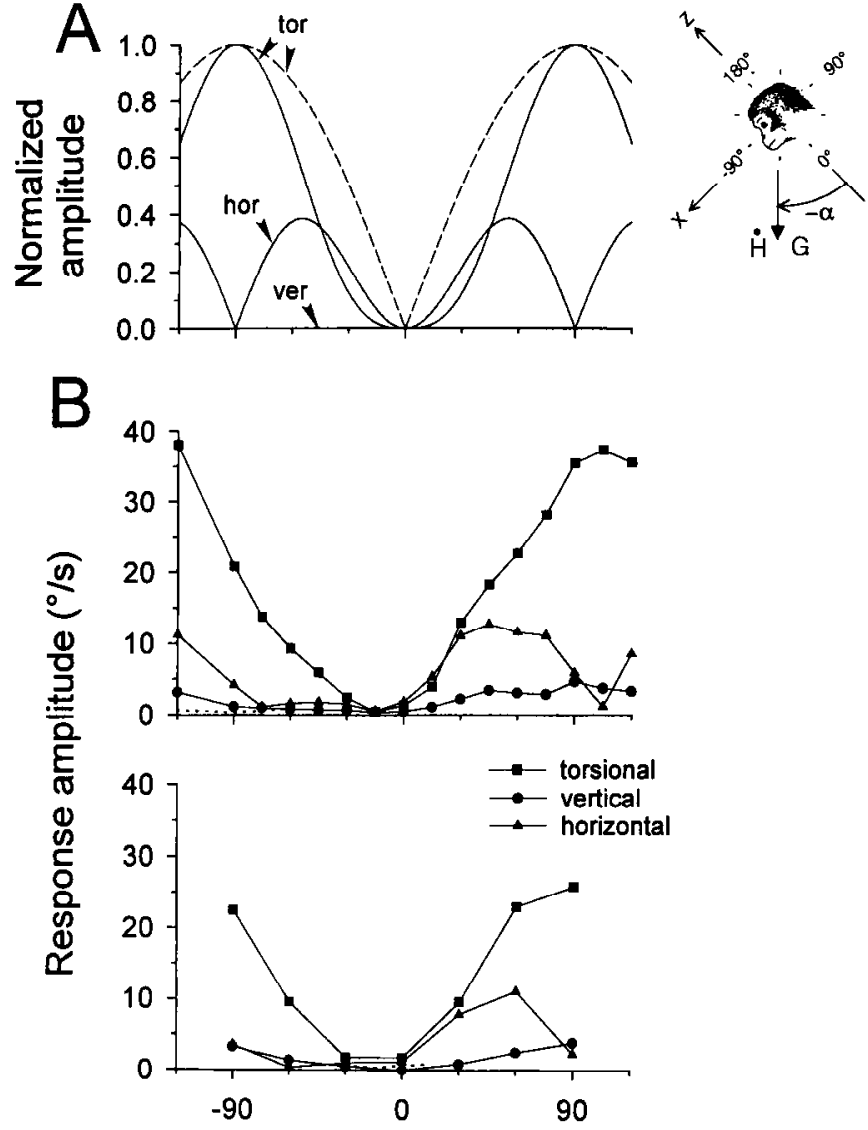

Stimulus angle $\alpha\left({ }^{\circ}\right)$

Figure 17. Low frequency VOR organization in the pitch plane (both lateral semicircular canals inactivated). A, Model simulations of slow phase eye velocity where vestibulo-ocular signals are either head-fixed (dashed lines) or transformed into a space-fixed incrtial representation (solid lines). In the head-fixed model, both horizontal and vertical response components are zero. In the space-fixed model, only vertical slow phase velocity is predicted to be zero. $B$, Horizontal (triangles), vertical (circles), and torsional (squares) slow phase eye velocity as a function of the orientation of the stimulus in the pitch plane (angle $\alpha$, see inset). Data from two animals recorded 7 months after semicircular canal inactivation $(0.01 \mathrm{~Hz})$.

crease beyond the prone position without ever reaching a peak up to the largest angle tested. Such strong asymmetric responses in prone/supine positions have been reported earlier (e.g., Minor and Goldberg, 1990).

\section{Discussion}

Semicircular canal afferents discharge in proportion to physical parameters related to the relative flow of the endolymphatic fluid in the semicircular canal ducts. The three pairs of semicircular canals provide an assembly of angular accelerometers that geometrically decompose any angular motion of the head into approximately three orthogonal components. Accordingly, semicircular canal primary afferents detect angular head acceleration within a noninertial head-fixed reference frame that is determined by the anatomical orientation of the semicircular canal ducts in the skull. The associated angular head motion in space needs still to be computed centrally based on this and other motion information. Complementary vestibular information regarding head angular velocity and position in space arises from the otolith system. Here we show that the central vestibular system does indeed compute angular motion of the head in space, presumably by correlating semicircular canal and otolith afferent information. These centrally computed inertial vestibular velocity signals dominate VOR responses at low frequencies.

Previous models of the vestibulo-ocular reflex have postulated two parallel pathways, one relaying semicircular canal afferent signals directly to central oculomotor circuitry and the other indirectly via a low-pass filter (identified by Raphan et al., 1979, as velocity storage). There are two assumptions inherent in this proposal. First, the semicircular canal inputs to these two pathways have so far been considered to be identical. Second, the two systems have been assumed to subserve similar functional roles, namely, gaze stabilization over a broad frequency range of head movements. According to this hypothesis, the two pathways supplement each other by enlarging the overall bandwidth of head velocity transduction in the vestibulo-ocular reflex. Our results strongly suggest, however, that the vestibular information coded in these two pathways is distinctly different. The first pathway carries short latency semicircular canal signals that represent instantaneous head angular velocity. These signals only depend on the relative orientation of the axis of rotation relative to the head and do not carry information about the spatial orientation of the axis of rotation in space. In the second pathway, however, semicircular canal signals are transformed relative to the head-fixed reference given by the semicircular canals such as to represent head angular velocity in space. Since these signals represent head velocity in space, we have called them "space-fixed" or "inertial" in contrast to the "head-fixed" angular velocity information provided by the semicircular canals. In the following, the evidence for space-referenced inertial vestibular signals will be briefly summarized before addressing the functional implications of our findings.

\section{Presence of inertial vestibular signals in the VOR}

It has been reported repeatedly in the past that during yaw rotation the late postrotatory responses align with gravity whenever the head is in a tilted position (Harris, 1987; Harris and Barnes, 1987; Angelaki and Anderson, 1991; Merfeld et al., 1991, 1993; Angelaki and Hess, 1994, 1995). In the majority of these studies, long duration time domain stimuli were used in order to demonstrate changes in postrotatory VOR which wcre only present late in the response due to the associated sluggish dynamics. These observations on the spatial orientation of the late postrotatory VOR have so far been interpreted as a simple expression of the fact that the central vestibular system detects the direction of absolute earth-vertical based on otolith information. It was not until recently when it was demonstrated that the spatial organization of the late postrotatory eye velocity is a basic three-dimensional feature of the VOR (Angelaki and Hess, 1994). Using fast tilts at the beginning of postrotatory responses, it was shown that slow phase eye velocity reorients relative to gravity independently of the plane of head rotation. It was thus concluded that all semicircular canal signals undergo a central transformation. Furthermore, it was proposed that spatial reorientation of the late postrotatory responses with gravity does not simply reflect a central representation of the earth-vertical but rather suggests the existence of an inertial vestibular system whose function is to detect head motion in space (Angelaki and Hess, 1994, 1995).

Our finding of a spatial transformation of not only lateral but also vertical semicircular canal afferent activity was at variance 
with previous reports. Earlier studies of the spatial organization of the vestibuloocular reflex had assumed that a gravity-based representation of vestibular signals was limited to the horizontal system (Raphan and Sturm, 1991). This assumption was based on the spatial response characteristics of optokinetic afternystagmus in different static head positions relative to gravity (Dai et al., 1991). Even though many properties of the velocity storage integrator seem to be shared by both the vestibular and optokinetic systems, this does not imply that all characteristics of optokinetic responses which are related to the velocity storage could a priori be extended as such to the vestibular system. During optokinetic stimulation, visual pathways are activated whose dependence on gravity signals has not been determined. If the gyroscopic properties of the vestibular system were limited to the "yaw axis eigenvector of the velocity storage integrator" as proposed by Raphan and colleagues, its manifestation in the oculomotor output would indeed only reflect a central representation of the spatial vertical (Dai et al., 1991). If, however, all semicircular canal signals were spatially transformed into a signal representing head motion in space, the functional ramifications of such processing would be much more intriguing. It would suggest a dynamic sensorimotor transformation, that is, the existence of an inertial vestibular system that uses activity from both scmicircular canal and otolith afferents in order to detect absolute head motion in space.

The results presented here demonstrate unequivocally that not only lateral but also vertical semicircular canal signals are centrally transformed such as to represent head motion in space. Two major features distinguish the experiments reported here from previous approaches to the problem of spatial coding of head velocity. First, the spatial organization of the VOR was investigated under static, steady-state conditions. Head position was kept constant relative to gravity in order to determine the influence of static otolith input on spatial processing of head angular velocity. Second, sinusoidal stimulation was used to independently assess frequency-specific characteristics in the spatial organization of vestibular information. The present results indeed revealed a frequency-specific spatial processing of vestibular head velocity signals in rhesus monkeys. While mid and high frequency VOR responses are found to be coded entirely relative to the head, low frequency VOR responses are largely determined by inertial vestibular signals, coding head motion in space.

The frequency analysis also revealed that it is exclusively the frequency-dependent relative changes in gain that determine the spatial organization of the VOR whereas the response phase of each component does not change relative to each other as a function of frequency (Fig. 9). Thus, the apparent differences in time constant of VOR components which accompanies the spatial reorganization of the late VOR responses (Hess and Angelaki, 1994, 1995; see also Fig. 6) is merely due to frequencydependent changes in gain and not due to changes in the associated response dynamics. These observations are suggestive of a system whose dynamics is due to low-pass filtering downstream of the spatial transformation (Fig. $5 C$ ). According to this hypothesis, inertial vestibular signals are computed before being relayed via a low-pass filter to the VOR.

The result that the two parallel vestibulo-ocular pathways carry distinct semicircular canal information challenges the widely accepted role of the velocity storage as a system that merely enhances low frequency VOR dynamics. The presence of lowpass filtered signals in the VOR can be regarded as the conse- quence of a mechanism that either extends the low frequency range or excludes high frequency signals from the system's output. Even though only the former goal has been considered so far as a functional explanation for the sluggish dynamics of velocity storage, our data suggest that the latter alternative is at least equally likely (Fig. $5 C$ ). Inertial vestibular signals must be excluded from mid and high frequency oculomotor control in order to avoid accumulation of retinal slip that would potentially jeopardize gaze stabilization. Nevertheless, the question still remains: why do inertial vestibular signals contribute at all to the VOR? Since these signals seem to be functionally coupled to motor control when both head and body are freely moving in space, the possibility exists that their presence in the VOR could be a consequence of immobilizing the head and neck as in the present experiments. If this is the case, the oculomotor contribution of inertial vestibular signals could be quite different under more natural head and body movements.

\section{Inertial detection of movement}

Inertial vestibular signal processing has been proposed as an important attribute of motor control and animal navigation several years ago (Barlow, 1964, 1966; Mayne, 1969, 1970, 1974). The original hypothesis was inspired by the development of man-made inertial guidance systems and supported by a number of psychophysical experiments. Since then, however, these fundamental ideas and their possible implications for vestibulo-motor control have had little impact on experimental work. During movement in a gravitational environment, correct detection of linear and angular components of motion in space are critical for orientation, as well as for motor control and coordination of posture and movement. The vestibular labyrinths are equipped with the necessary sensors that also constitute modern inertial guidance systems: the otolith organs represent a set of threedimensional linear accelerometers that detect gravitational and translational components associated with any head movement. This information is supplemented by the semicircular canals that represent a set of three-dimensional angular accelerometers with built-in integrators that accurately detect head angular velocity. The complexity of inertial navigation systems arises when these two independent sets of signals are to be combined in order to compute motion in space. The computational problems can be summarized by the following two goals. (1) Translational and gravitational components of linear accelerations which are physically equivalent and can not be distinguished by any physical or biological linear accelerometer must be independently computed. (2) Absolute angular position and velocity in space must be determined. These two goals are not independent since detection of absolute angular position and velocity in space is coupled to the correct assessment of gravitational accelerations. Translational accelerations, on the other hand, must be separated and interpreted as such in order to evoke appropriate cognitive and motor actions. Even though it is presently unknown to what extent these goals are realized in the vestibulo-motor system, our data suggest the existence of an inertial vestibular system as a computational center which uses signals from the two sets of linear and angular motion sensors to centrally compute absolute angular motion in space.

We have previously argued that inertial coding of head motion in space would be advantageous for head movement control, as well as motor coordination of gaze, head and body posture (Angelaki and Hess, 1994, 1995). Inertial vestibular signals could represent the backbone in computational processes related to dy- 
namic control of posture and equilibrium, as well as in the coordination of eye, head and gaze in space. Even though the main function seems not to be oculomotor, inertial vestibular signals could be expressed in the oculomotor system as part of a gaze control mechanism that is involved in spatial orientation during head and body movements. A better understanding of its functional significance requires further investigations in head-free and freely moving subjects.

\section{References}

Angelaki DE, Anderson JH (1991) The horizontal vestibulo-ocular reflex during linear acceleration in the frontal plane of the cat. Exp Brain Res 86:40-46.

Angelaki DE, Hess BJM (1994) Inertial representation of angular motion in the vestibular system of rhesus monkeys. I. Vestibulo-ocular reflex. J Neurophysiol 71:1222-1249.

Angelaki DE, Hess BJM (1995) Inertial representation of angular motion in the vestibular system of rhesus monkeys. II. Otolith-controlled transformation that depends on an intact cerebellar nodulus. J Neurophysiol 73:1729-1751.

Angelaki DE, Hess BJM, Suzuki I-J (1994) Spatiotemporal VOR adaptation after semicircular canal inactivation. Soc Neurosci Abstr 20: 1194.

Barlow JS (1964) Inertial navigation as a basis for animal navigation. J Theor Biol 6:76-117.

Barlow JS (1966) Inertial navigation in relation to animal navigation. $J$ Inst Navigation 302-316.

Cohen B, Matsuo V, Raphan Th (1977) Quantitative analysis of the velocity characteristics of optokinetic nystagmus and optokinetic afternystagmus. J Physiol (Lond) 270:321-344.

Dai M, Raphan T, Cohen B (1991) Spatial orientation of the vestibular system: dependence of optokinetic afternystagmus on gravity. J Neurophysiol 66:1422-1439.

Ewald JR (1892) Physiologische Untersuchugen uber dass Endorgan des Nervus oclavus. Wiesbaden: Bergmann.

Fernández C, Goldberg JM (1971) Physiology of peripheral neurons innervating semicircular canals of the squirrel monkey. II. Response to sinusoidal stimulation and dynamics of peripheral vestibular system. J Neurophysiol 34:661-675.

Harris LR (1987) Vestibular and optokinetic eye movements evoked in the cat by rotation about a tilted axis. Exp Brain Res 66:522-532.
Harris LR, Barnes GR (1987) Orientation of vestibular nystagmus is modified by head tilt. In: The vestibular system: neurophysiology and clinical research (Graham MD, Kemink JL, eds), pp 539-549. New York: Raven.

Haustein W (1989) Considerations on Listing's law and primary position by means of a matrix description of eye position control. Biol Cybern 60:411-420.

Hess BJM, Van Opstal AJ, Straumann D, Hepp K (1992) Calibration of three-dimensional eye position using search coil signals in the rhesus monkey. Vision Res 32:1647-1654.

Mayne R (1969) The biological inertial system. Proc IEEE Resources Round-up, Region 6, pp 209-222. Phoenix: Arizona.

Mayne R (1970) The analogy of the vestibular organs to an inertial guidance system. Proc Ninth Int Congress, Oto-Rhino-Laryngology, Mexico City, 1969. Excerpta Med Amsterdam 407-415.

Mayne R (1974) A systems concept of the vestibular organs. In: Handbook of sensory physiology vestibular system (Kornhuber $\mathbf{H H}$, ed), pp 493-580. Ncw York: Springer.

Merfeld DM, Young LR, Paige GD, Tomko DL (1993) Three-dimensional eye movements of squirrel monkeys following postrotatory tilt. J Vest Res 3:123-139.

Merfeld DM, Young LR, Tomko DL, Paige GD (1991) Spatial orientation of VOR to combined vestibular stimuli in squirrel monkeys. Acta Otolaryngol Suppl 481:287-292.

Minor LB, Goldberg JM (1990) Influence of static head position on the horizontal nystagmus evoked by caloric, rotational and optokinetic stimulation in the squirrel monkey. Exp Brain Res 82:1-13.

Money KE, Scott JW (1962) Functions of separate sensory receptors of non-auditory labyrinth of the cat. Am J Physiol 202:1211-1220.

Raphan T, Sturm D (1991) Modeling the spatiotemporal organization of velocity storage in the vestibuloocular reftex by optokinetic studies. J Neurophysiol 66:1410-1421.

Raphan T, Matsuo V, Cohen B (1979) Velocity storage in the vestibuloocular reflex arc (VOR). Exp Brain Res 35:229-248.

Raphan T, Wcarne S, Cohen B (1994) Static and dynamic effects on gravito-inertial acceleration (GIA) on spatial orientation of velocity storage. Soc Neurosci Abstr 20:1195.

Solomon D, Cohen B (1992) Stabilization of gaze during circular locomotion in darkness. II. Contribution of velocity storage to compensatory eye and head nystagmus in the running monkey. J Neurophysiol 67:1158-1170.

Suzuki J-I, Kodama A, Cohen B, Henn V (1991) Canal-plugging in the rhesus monkey: A tool to study the contribution of individual canals to nystagmus generation. Acta Otolaryngol Suppl 481:91-93. 